\title{
Nonlinear Multivalued Periodic Systems
}

\author{
${\text { Leszek Gasiński }{ }^{1} \text { - Nikolaos S. Papageorgiou }}^{2}$
}

Received: 17 November 2017 / Revised: 8 May 2018 / Published online: 14 June 2018

(C) The Author(s) 2018

\begin{abstract}
We consider a first-order periodic system involving a time-dependent maximal monotone map, a subdifferential term, and a multivalued perturbation $F(t, x)$. We prove existence theorems for the "convex" problem (that is, $F$ is convex valued and for the "nonconvex" problem (that is, $F$ is nonconvex valued). Also, we establish the existence of extremal trajectories (that is, solutions when the multivalued perturbation $F(t, x)$ is replaced by ext $F(t, x)$, the extreme points of $F(t, x))$. Also, we show that every solution of the convex problem can be approximated uniformly by certain extremal trajectories ("strong relaxation" theorem). Finally, we illustrate our result by examining a nonlinear periodic feedback control system.
\end{abstract}

Keywords Convex problem $\cdot$ Nonconvex problem $\cdot$ Extremal trajectories $\cdot$ Strong relaxation $\cdot$ Maximal monotone map $\cdot$ Control system

Mathematics Subject Classification (2010) $34 \mathrm{~A} 34 \cdot 34 \mathrm{~B} 15 \cdot 47 \mathrm{~N} 20$

The research was supported by the National Science Center of Poland under Project No. 2015/19/B/ST1/01169.

Leszek Gasiński

Leszek.Gasinski@ii.uj.edu.pl

Nikolaos S. Papageorgiou

npapg@math.ntua.gr

1 Faculty of Mathematics and Computer Science, ul Łojasiewicza 6, 30-348 Kraków, Poland

2 Department of Mathematics Zografou Campus, National Technical University, 15780 Athens, Greece 


\section{Introduction}

In the present work, we study the following nonlinear multivalued periodic system:

$$
\left\{\begin{array}{l}
-u^{\prime}(t) \in A(t, u(t))+\partial \varphi(u(t))+F(t, u(t)) \quad \text { for a.a. } t \in T=[0, b] \\
u(0)=u(b) .
\end{array}\right.
$$

In this problem, $A: T \times \mathbb{R}^{N} \longrightarrow 2^{\mathbb{R}^{N}} \backslash \emptyset$ is a multivalued map which is maximal monotone in $x \in \mathbb{R}^{N}, \varphi \in \Gamma_{0}\left(\mathbb{R}^{N}\right)$ (the cone of lower semicontinuous, convex, proper functions; see Section 2) with $\partial \varphi$ being the subdifferential in the sense of convex analysis and $F: T \times \mathbb{R}^{N} \longrightarrow 2^{\mathbb{R}^{N}} \backslash \emptyset$ is a multivalued perturbation. We prove existence theorems for problem (1.1) when $F$ is convex valued ("convex problem") and when $F$ is nonconvex valued ("nonconvex problem"). We also show the existence of extremal trajectories, that is, solutions of Eq. 1.1 when $F(t, x)$ is replaced by ext $F(t, x)$ (the set of extreme points of $F(t, x)$ ). Moreover, we show that every solution of the convex problem can be approximated in the $C\left(T ; \mathbb{R}^{N}\right.$ )-norm by certain extremal trajectories ("strong relaxation" theorem). An example of a feedback periodic control system illustrate our results.

Our work here is related to those of Frigon [5] and Qin and Xue [14]. In Frigon [5], $\varphi \equiv 0$ and $A$ is time-independent with $D(A) \neq \mathbb{R}^{N}$. The author proves existence theorems for both the convex and nonconvex problems using the notion of $L^{p}$-solution tube. Qin and Xue [14] assume that that $A$ is time-independent and is a positive definite $N \times N$-matrix. Also, they assume that $\varphi: \mathbb{R}^{N} \longrightarrow \mathbb{R}$ is continuous convex. They deal with the convex and nonconvex problems and also address the question of existence of extremal trajectories. Finally, we mention the work of Bader and Papageorgiou [1], where $A \equiv 0$, but the inclusion takes place in the context of a general separable Hilbert space.

\section{Mathematical Background-Hypotheses}

Our approach is based on tools from multivalued analysis (see Hu-Papageorgiou [11]) and from the theory of nonlinear operators of monotone type (see Gasiński-Papageorgiou [7] and Zeidler [15]).

Let $(\Omega, \Sigma)$ be a measurable space and $X$ a separable Banach space. We use the following notation:

$$
\begin{aligned}
P_{f(c)}(X) & =\{A \subseteq X: A \text { is nonempty, closed (and convex) }\} \\
P_{(w) k(c)}(X) & =\{A \subseteq X: A \text { is nonempty, (w-)compact (and convex) }\} .
\end{aligned}
$$

A multifunction (set-valued function), $F: \Omega \longrightarrow 2^{X} \backslash \emptyset$ is said to be "graph measurable," if $\operatorname{Gr} F \in \Sigma \otimes \mathcal{B}(X)$, where

$$
\operatorname{Gr} F=\{(\omega, x) \in \Omega \times X: x \in F(\omega)\} \in \Sigma \otimes \mathcal{B}(X),
$$

with $\mathcal{B}(X)$ being the Borel $\sigma$-field of $X$. If $\Sigma=\widehat{\Sigma}$, the universal $\sigma$-field (this is the case if there is a $\sigma$-finite measure $\mu$ on $\Sigma$ and $\Sigma$ is $\mu$-complete), then the Yankov-von Neumann-Aumann selection theorem (see Hu and Papageorgiou [11, p.158] or Gasiński and Papageorgiou [7, p. 906]) says that every graph measurable multifunction $F: \Omega \longrightarrow 2^{X} \backslash \emptyset$ admits a measurable selection, that is, there exists a $\Sigma$-measurable function $f: \Omega \longrightarrow X$ such that $f(\omega) \in F(\omega)$ for all $\omega \in \Omega$. In fact, there is a whole sequence $\left\{f_{n}: \Omega \longrightarrow X\right\}_{n} \geqslant 1$ of measurable selections such that

$$
F(\omega) \subseteq{\overline{\left\{f_{n}(\omega)\right\}_{n}}}_{n 1} \quad \forall \omega \in \Omega .
$$


The result is true if the separable Banach space $X$ is replaced by a Souslin space. Recall that a Souslin space is always separable but need not be metrizable. For example, if $X^{*}$ is the dual of a separable Banach space and it is equipped with the $w^{*}$-topology, then it is a nonmetrizable Souslin space.

A multifunction $F: \Omega \longrightarrow P_{f}(X)$ is said to be "measurable", if for all $x \in X$, the function

$$
\omega \longmapsto d(x, F(\omega))=\inf _{u \in F(\omega)}\|x-u\|
$$

is $\Sigma$-measurable. This is equivalent to saying that for every open set $U \subseteq X$, the set

$$
F^{-}(U)=\{\omega \in \Omega: F(\omega) \cap U \neq \emptyset\} \in \Sigma .
$$

A measurable multifunction $F: \Omega \longrightarrow P_{f}(X)$ is graph measurable. The converse is true if there is a $\sigma$-finite, complete measure defined on $\Sigma$.

Now, let $(\Omega, \Sigma, \mu)$ be a $\sigma$-finite measure space and $X$ a separable Banach space. Given $1 \leqslant p \leqslant+\infty$ and a graph measurable multifunction $F: \Omega \longrightarrow 2^{X} \backslash \emptyset$, we define

$$
S_{F}^{p}=\left\{f \in L^{p}(\Omega ; X): f(\omega) \in F(\omega) \mu-\text { a.e. }\right\} .
$$

A straightforward application of the Yankov-von Neumann-Aumann selection theorem, reveals that " $S_{F}^{p} \neq \emptyset$ if and only if $\omega \longmapsto \inf \{\|u\|: u \in F(\omega)\}$ belongs in $L^{p}(\Omega)$." This set is "decomposable," that is, if $\left(A, f_{1}, f_{2}\right) \in \Sigma \times S_{F}^{p} \times S_{F}^{p}$, then

$$
\chi_{A} f_{1}+\chi_{\Omega \backslash A} f_{2} \in S_{F}^{p} .
$$

Here, for $C \in \Sigma, \chi_{C}$ denotes the characteristic function of $C$, hence

$$
\chi_{C}(\omega)=\left\{\begin{array}{l}
1 \text { if } \omega \in C, \\
0 \text { if } \omega \in \Omega \backslash C .
\end{array}\right.
$$

Since $\chi_{\omega \backslash C}=1-\chi_{C}$, we see that the notion of decomposability formally looks very similar to that of convexity, only now the coefficients in the linear combination are functions. In fact, decomposable sets exhibit properties which are similar the those of convex sets (see Hu and Papageorgiou [11, Section 2.3]).

Suppose that $Y$ and $Z$ are Hausdorff topological spaces and let $G: Y \longrightarrow 2^{Z} \backslash \emptyset$ be a multifunction. We say that $G$ is "upper semicontinuous" if for every open set $U \subseteq Z$, the set $G^{+}(U)=\{y \in Y: G(y) \subseteq U\}$ is open. We say that $G$ is "lower semicontinuous" if for every open set $U \subseteq Z$, the set $G^{-}(U)=\{y \in Y: G(y) \cap U \neq \emptyset\}$ is open.

An upper semicontinuous multifunction with closed values has closed graph. The converse is true, if $G$ is locally compact (that is, for every $x \in X$, there exists a neighborhood $U$ of $x$ such that $\overline{G(U)} \in P_{k}(Z)$ ). If $Z$ is a metric space, then $G$ is lower semicontinuous if and only if for all $z \in Z, y \longmapsto d_{Z}(z, G(y))=\inf _{v \in G(y)} d_{Z}(z, v)$ is an upper semicontinuous $\mathbb{R}_{+}$-valued function (here, $d_{Z}$ denotes the metric of $Z$ ).

For a metric space $Z$ (with metric $d_{Z}$ ) on $P_{f}(Z)$, we can define a generalized metric, known as the "Hausdorff metric", by setting

$$
h(C, E)=\sup _{u \in Z} \mid d_{Z}\left(u, C\left|-d_{Z}(u, E)\right| \quad \forall C, E \in P_{f}(Z) .\right.
$$

If $Z$ is complete, then so is $\left(P_{f}(Z), h\right)$. A multifunction $G: Y \longrightarrow P_{f}(Z)$ is said to be " $h$-continuous", if it is continuous from the Hausdorff topological space $Y$ into the metric space $\left(P_{f}(Z), h\right)$.

Suppose that $V$ is a Banach space and $C \subseteq V$ is nonempty. We set

$$
|C|=\sup _{u \in C}\|u\|_{V}
$$


Also, if $\left\{C_{n}\right\}_{n} \geqslant 1 \subseteq 2^{V} \backslash \emptyset$, we define

$$
\begin{aligned}
\liminf _{n \rightarrow+\infty} C_{n} & =\left\{u \in V: u=\lim _{n \rightarrow+\infty} u_{n}, u_{n} \in C_{n} \text { for all } n \in \mathbb{N}\right\} \\
& =\left\{u \in V: \lim _{n \rightarrow+\infty} d\left(u, C_{n}\right)=0\right\}
\end{aligned}
$$

and

$$
w-\limsup _{n \rightarrow+\infty} C_{n}=\left\{u \in V: u=w \lim _{k \rightarrow+\infty} u_{n_{k}}, u_{n_{k}} \in C_{n_{k}}, n_{1}<n_{2}<\ldots\right\} .
$$

Next, let $X$ be a reflexive Banach space and $X^{*}$ its topological dual. By $\langle\cdot, \cdot\rangle$, we denote the duality brackets for the pair $\left(X^{*}, X\right)$. A multivalued map $A: X \supseteq D \longrightarrow 2^{X^{*}}$ is said to be "monotone", if

$$
\left\langle x^{*}-u^{*}, x-u\right\rangle \geqslant 0 \quad \forall\left(x, x^{*}\right),\left(u, u^{*}\right) \in \mathrm{Gr} A .
$$

Here, $D=\{x \in X: A(x) \neq \emptyset\}$, the "domain" of $A$.

We say that a monotone map is "strictly monotone," if

$$
\left\langle x^{*}-u^{*}, x-y\right\rangle=0 \Longrightarrow x=y .
$$

The monotone map $A: X \supseteq D \longrightarrow 2^{X^{*}}$ is "maximal monotone," if

$$
\left\langle x^{*}-u^{*}, x-u\right\rangle \geqslant 0 \text { for all }\left(u, u^{*}\right) \in \operatorname{Gr} A \Longrightarrow\left(x, x^{*}\right) \in \operatorname{Gr} A .
$$

This means that $\mathrm{Gr} A$ is maximal with respect to inclusion among the graphs of monotone maps. It is easy to see that, if $A: X \supseteq D \longrightarrow 2^{X^{*}}$ is maximal monotone, then $\mathrm{Gr} A$ is sequentially closed in $X_{w} \times X^{*}$ and in $X \times X_{w}^{*}$ (here, by $X_{w}$ and $X_{w}^{*}$, we denote the spaces $X$ and $X^{*}$, respectively, furnished with the weak topology). If $A$ is maximal monotone, then for every $x \in D, A(x) \in P_{f c}\left(X^{*}\right)$.

For a maximal monotone map $A: X \longrightarrow 2^{X^{*}}$, we define

$$
A^{0}(x)=\left\{x^{*} \in A(x):\left\|x^{*}\right\|_{*}=\inf _{u^{*} \in A(x)}\left\|u^{*}\right\|_{*}\right\} \quad \forall x \in D .
$$

Since for every $x \in D, A(x) \in P_{f c}\left(X^{*}\right)$ and $X$ is reflexive, then $A^{0}(x) \neq \emptyset$. Moreover, if $X^{*}$ is strictly convex, then $A^{0}$ is single-valued. The map $A^{0}$ is called the "minimal section" of $A$.

The "duality map" $\mathcal{F}: X \longrightarrow 2^{X^{*}}$ is defined by

$$
\mathcal{F}(u)=\left\{u^{*} \in X^{*}:\left\langle u^{*}, u\right\rangle=\|u\|^{2}=\left\|u^{*}\right\|_{*}^{2}\right\} \quad \forall u \in X .
$$

The Hahn-Banach theorem implies that $\mathcal{F}$ has nonempty values. In fact, the duality map is defined for any Banach space. However, its properties strongly depend on the geometry of the Banach space $X$. In particular, if $X$ and $X^{*}$ are both locally uniformly convex, then $\mathcal{F}$ is single-valued and a homeomorphism.

By $\Gamma_{0}(X)$, we denote the cone of all functions $\varphi: X \rightarrow \overline{\mathbb{R}}=\mathbb{R} \cup\{+\infty\}$ which are lower semicontinuous, convex, and proper (that is, $\operatorname{dom} \varphi=\{x \in X: \varphi(x)<\infty\}$ (the effective domain of $\varphi$ ) is nonempty). By $\partial \varphi: X \longrightarrow 2^{X^{*}}$, we denote the subdifferential of $\varphi$ in the sense of convex analysis, that is

$$
\partial \varphi(u)=\left\{u^{*} \in X^{*}:\left\langle u^{*}, h\right\rangle \leqslant \varphi(u+h)-\varphi(u) \text { for all } h \in X\right\} .
$$

If $\varphi$ is continuous at $u$, then $\partial \varphi(u) \neq \varnothing$. If $\varphi$ is Gâteaux differentiable at $u$, then $\partial \varphi(u)=$ $\left\{\varphi_{G}^{\prime}(u)\right\}\left(\varphi_{G}^{\prime}(u)\right.$ denotes the Gâteaux derivative of $\varphi$ at $\left.u\right)$. The map $\partial \varphi: X \longrightarrow 2^{x^{*}}$ is maximal monotone. 
By $L_{w}^{1}\left(T ; \mathbb{R}^{N}\right)$, we denote the Lebesgue space $L^{1}\left(T ; \mathbb{R}^{N}\right)$ equipped with the weak norm

$$
\|u\|_{w}=\sup _{0 \leqslant s \leqslant t \leqslant b}\left|\int_{s}^{t} u(\tau) d \tau\right| \quad \forall u \in L^{1}\left(T ; \mathbb{R}^{N}\right)
$$

or equivalently by

$$
\|u\|_{w}=\sup _{0 \leqslant t \leqslant b}\left|\int_{0}^{t} u(\tau) d \tau\right| .
$$

This norm is equivalent to the Pettis norm (see Egghe [4]).

The hypotheses on the map $A$ and on the function $\varphi$ are the following:

$H(A): A: T \times \mathbb{R}^{N} \longrightarrow 2^{\mathbb{R}^{N}} \backslash \emptyset$ is a multifunction such that $0 \in A(t, 0)$ for all $t \in T$ and

(i) $(t, x) \longrightarrow A(t, x)$ is graph measurable and for all $t \in T, x \longmapsto A(t, x)$ is maximal monotone.

(ii) There exist two continuous functions $\eta: T \longrightarrow \mathbb{R}^{N}$ and $l: \mathbb{R}_{+} \longrightarrow \mathbb{R}_{+}$such that

$$
\left(h_{1}-h_{2}, u_{1}-u_{2}\right)_{\mathbb{R}^{N}} \geqslant-|\eta(t)-\eta(s)|\left|u_{1}-u_{2}\right| l\left(\max \left\{\left|u_{1}\right|,\left|u_{2}\right|\right\}\right)
$$

for all $0 \leqslant s \leqslant t \leqslant b$ and all $\left(u_{1}, h_{1}\right) \in \operatorname{Gr} A(t, \cdot),\left(u_{2}, h_{2}\right) \in \operatorname{Gr} A(s, \cdot)$.

(iii) For every $r>0$, there exists $a_{r} \in L^{2}(T)$ such that

$$
|A(t, x)| \leqslant a_{r}(t) \quad \text { for a.a. } t \in T, \text { all }|x| \leqslant r
$$

and for all $u \in L^{2}\left(T ; \mathbb{R}^{N}\right), t \longmapsto A^{0}(t, u(t))$ belongs in $L^{2}\left(T ; \mathbb{R}^{N}\right)$.

Remark 2.1 Hypothesis $H(A)(i)$ implies that

$$
A(t, x) \in P_{k c}\left(\mathbb{R}^{N}\right) \quad \forall(t, x) \in T \times \mathbb{R}^{N} .
$$

Hypothesis $H(A)(i i)$ imposes restrictions on the $t$-dependence of $A$ and permits the use of the theory of evolution equations involving time-dependent operators (see Pavel [13]).

$$
\begin{aligned}
& H(\varphi): \varphi \in \Gamma_{0}\left(\mathbb{R}^{N}\right) \text { with } 0 \in D(\partial \varphi) \text { and } \\
& D(\partial \varphi)=\text { intdom } \varphi \text { or } \varphi \text { is bounded above on bounded sets. }
\end{aligned}
$$

Remark 2.2 Both conditions in the above hypothesis imply that $u \longmapsto \partial \varphi(u)$ is bounded (that is, maps bounded sets to bounded sets). The following function $\varphi$ satisfies the first condition, namely that $D(\partial \varphi)=\operatorname{intdom} \varphi$

$$
\varphi(u)= \begin{cases}-\left(1-|u|^{2}\right)^{\frac{1}{2}} & \text { if }|u| \leqslant 1, \\ +\infty & \text { otherwise }\end{cases}
$$

for all $u \in \mathbb{R}^{N}$.

Next, we prove a result which we will need in the sequel and which is of independent interest. For this reason, it is formulated in a more general setting than the one in which it will be used in this paper. We mention that the result is known for Hilbert spaces (see Brézis $[3$, p. 25]).

So, as before, let $X$ be a reflexive Banach space and $X^{*}$ its topological dual. By $|\cdot|$ and $|\cdot|_{*}$, we denote the norm on $X$ and $X^{*}$ respectively and by $\langle\cdot, \cdot\rangle$ the duality brackets for the pair $\left(X^{*}, X\right)$. Let $A: X \longrightarrow 2^{X^{*}}$ be a maximal monotone map with $0 \in A(0)$. On account of the Troyanski renorming theorem (see, e.g., Gasiński-Papageorgiou [7, p. 911]), without any loss of generality, we may assume that both $X$ and $X^{*}$ are locally uniformly convex. 
We know that the duality map $\mathcal{F}: X \longrightarrow X^{*}$ is single-valued and a homeomorphism. We introduce the lifting (realization) of $A$ on the dual pair $\left(L^{p^{\prime}}\left(T ; X^{*}\right), L^{p}(T ; X)\right), 1 \leqslant p<$ $+\infty, 1<p^{\prime} \leqslant+\infty, \frac{1}{p}+\frac{1}{p^{\prime}}=1$ (recall that $L^{p}(T ; X)^{*}=L^{p^{\prime}}\left(T ; X^{*}\right)$; see Gasiński and Papageorgiou [7, p. 129]), $\mathcal{A}: L^{p}(T ; X) \longrightarrow L^{p^{\prime}}\left(T ; X^{*}\right)$ defined by

$$
\mathcal{A}(u)=\left\{u^{*} \in L^{p^{\prime}}\left(T ; X^{*}\right): u^{*}(t) \in A(u(t)) \text { for a.a. } t \in T\right\}
$$

for all $u \in D(\mathcal{A})=\left\{u \in L^{p}(T ; X): S_{A(u(\cdot))}^{p^{\prime}} \neq \emptyset\right\}$.

In what follows by $((\cdot, \cdot))$, we denote the duality brackets for the pair of spaces $\left(L^{p^{\prime}}\left(T ; X^{*}\right), L^{p}(T ; X)\right)$. So, we have

$$
((h, u))=\int_{0}^{b}\langle h(t), u(t)\rangle d t \quad \forall h \in L^{p^{\prime}}\left(T ; X^{*}\right), u \in L^{p}(T ; X) .
$$

Lemma 2.3 If $A: X \longrightarrow 2^{X^{*}}$ is a maximal monotone map with $0 \in A(0)$, then $\mathcal{A}: L^{p}(T ; X) \longrightarrow 2^{L^{p^{\prime}}\left(T ; X^{*}\right)}$ is maximal monotone.

Proof Let $\vartheta: L^{p}(T ; X) \longrightarrow L^{p^{\prime}}\left(T ; X^{*}\right)$ be the map defined by

$$
\vartheta(u)(\cdot)=|u(\cdot)|^{p-2} \mathcal{F}(u(\cdot)) \quad \forall u \in L^{p}(T ; X) .
$$

Evidently, $\vartheta$ is continuous and strictly monotone, thus, maximal monotone too (see Gasiński and Papageorgiou [7, p. 310]).

Claim $R(\mathcal{A}+\vartheta)=L^{p^{\prime}}\left(T ; X^{*}\right)$ (that is, $\mathcal{A}+\vartheta$ is surjective).

Let $h \in L^{p^{\prime}}\left(T ; X^{*}\right)$ and consider the multifunction $K: T \longrightarrow 2^{X}$ defined by

$$
K(t)=\left\{x \in X: h(t) \in A(x)+|x|^{p-2} \mathcal{F}(x)\right\} .
$$

The map $x \longmapsto A(x)+|x|^{p-2} \mathcal{F}$ is maximal monotone and coercive. Hence, it is surjective (see Gasiński and Papageorgiou [7, p. 336]). Therefore, $K(t) \neq \emptyset$ for all $t \in T \backslash N$, with $N$ being Lebesgue-null. On this exceptional null set, we set $K(t)=\{0\}$. Note that

$$
\text { Gr } K=\left\{(t, x) \in T \times X:\left(x, h(t)-|x|^{p-2} \mathcal{F}(x)\right) \in \text { Gr } A\right\} .
$$

We know that the maximal monotonicity of $A$ implies that $\operatorname{Gr} A \subseteq X \times X^{*}$ is closed. Moreover, the map $\xi: T \times X \longrightarrow X \times X^{*}$ defined by

$$
\xi(t, x)=\left(x, h(t)-|x|^{p-2} \mathcal{F}(x)\right)
$$

is a Carathéodory mao, that is, for all $x \in X, t \longmapsto \xi(t, x)$ is measurable, while for almost all $t \in T, x \longmapsto \xi(t, x)$ is continuous. We know that $\xi$ is jointly measurable (see $\mathrm{Hu}$ and Papageorgiou [11, p. 142]). Hence

$$
\xi^{-1}(\operatorname{Gr} A)=\operatorname{Gr} K \in \mathcal{L}_{T} \otimes \mathcal{B}(X),
$$

with $\mathcal{L}_{T}$ being the Lebesgue $\sigma$-field on $T$ and $\mathcal{B}(X)$ the Borel $\sigma$-field of $X$. Invoking the Yankov-von Neumann-Aumann selection theorem, we can find a measurable map $u: T \longrightarrow$ $X$ such that

$$
u(t) \in K(t) \quad \forall t \in T,
$$

so

$$
h(t) \in A(u(t))+|u(t)|^{p-2} \mathcal{F}(u(t)) \quad \text { for a.a. } t \in T .
$$

We act with $u(t) \in X$ and recall that by hypothesis $0 \in A(0)$, we obtain

$$
|u(t)|^{p-1} \leqslant|h(t)| \quad \text { for a.a. } t \in T,
$$


thus, $u \in L^{p}(T ; X)$ (recall that $\left.p-1=\frac{p}{p^{\prime}}, h \in L^{p^{\prime}}\left(T ; X^{*}\right)\right)$ and $h \in \mathcal{A}(u)+\vartheta(u)$.

Since $h \in L^{p^{\prime}}\left(T ; X^{*}\right)$ is arbitrary, we conclude that

$$
R(\mathcal{A}+\vartheta)=L^{p^{\prime}}\left(T ; X^{*}\right) .
$$

This proves the Claim.

Evidently the map $\mathcal{A}$ is monotone. We will show that in fact it is maximal monotone. To this end, suppose that $(v, g) \in L^{p}(T ; X) \times L^{p^{\prime}}\left(T ; X^{*}\right)$ and assume that

$$
((h-g, u-v))=\int_{0}^{b}\langle h(t)-g(t), u(t)-v(t)\rangle d t \geqslant 0 \quad \forall(u, h) \in \operatorname{Gr} \mathcal{A} .
$$

On account of the Claim, we can find $\left(u_{1}, h_{1}\right) \in \operatorname{Gr} \mathcal{A}$ such that

$$
h_{1}+\vartheta\left(u_{1}\right)=g+\vartheta(v) \text {. }
$$

We return to Eq. 2.1 and choose $(u, h)=\left(u_{1}, h_{1}\right)$. Then, using Eq. 2.2, we have

$$
0 \leqslant \int_{0}^{b}\left\langle g+\vartheta(v)-\vartheta\left(u_{1}\right)-g, u_{1}-v\right\rangle d t=\int_{0}^{b}\left\langle\vartheta(v)-\vartheta\left(u_{1}\right), u_{1}-v\right\rangle d t,
$$

so $u_{1}=v$ (since $\vartheta$ is strictly monotone), thus, $(v, g) \in \operatorname{Gr} A$ and hence $\mathcal{A}$ is maximal monotone.

\section{The Convex Problem}

In this section, we prove an existence theorem for problem (1.1) when the multivalued perturbation $F$ is convex valued.

The precise hypotheses on $F$ are the following:

$H(F)_{1}: F: T \times \mathbb{R}^{N} \longrightarrow P_{k c}\left(\mathbb{R}^{N}\right)$ is a multifunction such that

(i) For all $x \in \mathbb{R}^{N}, t \longrightarrow F(t, x)$ is graph measurable.

(ii) For almost all $t \in T$, Gr $F(t, \cdot) \in \mathbb{R}^{N} \times \mathbb{R}^{N}$ is closed.

(iii) There exist $M>0$ and $\widehat{a}_{M} \in L^{2}(T)$ such that

$$
\begin{gathered}
0 \leqslant(h, x)_{\mathbb{R}^{N}} \quad \text { for a.a. } t \in T, \text { all }|x|=M, h \in F(t, x), \\
|F(t, x)| \leqslant \widehat{a}_{M}(t) \quad \text { for a.a. } t \in T,|x| \leqslant M .
\end{gathered}
$$

Remark 3.1 Hypotheses $H(F)_{1}(i)$ and (ii) do not imply joint measurability of $F$ (see $\mathrm{Hu}$ and Papageorgiou [11, p. 226]). So, for $u: T \longrightarrow \mathbb{R}^{N}$ measurable, $|u(t)| \leqslant M$ almost everywhere on $T$, it is not a priori clear that $S_{F(\cdot, u(\cdot))}^{2} \neq \emptyset$. To show the nonemptiness of this set, we argue as follows. Let $\left\{s_{n}\right\}_{n} \geqslant 1$ be a sequence of step functions such that $s_{n}(t) \longrightarrow u(t)$ for almost all $t \in T$ and $\left|s_{n}(t)\right| \leqslant|u(t)|$ for almost all $t \in T$, all $n \in \mathbb{N}$. Then, hypothesis $H(F)_{1}$ implies that for every $n \in \mathbb{N}, t \longmapsto F\left(t, s_{n}(t)\right)$ is measurable and so by the Yankov-von Neumann-Aumann selection theorem, we can find $h_{n}: T \longrightarrow \mathbb{R}^{N}$ measurable such that

$$
h_{n}(t) \in F\left(t, s_{n}(t)\right) \text { for a.a. } t \in T \text {, all } n \geqslant \mathbb{N} \text {, }
$$

so the sequence $\left\{h_{n}\right\}_{n} \geqslant 1 \subseteq L^{2}\left(T ; \mathbb{R}^{N}\right)$ is bounded.

Therefore, by passing to a subsequence if necessary, we may assume that

$$
h_{n} \stackrel{w}{\longrightarrow} h \quad \text { in } L^{2}\left(T ; \mathbb{R}^{N}\right),
$$


with $h \in L^{2}\left(T ; \mathbb{R}^{N}\right)$. Invoking Proposition 3.9 of $\mathrm{Hu}$ and Papageorgiou [11, p. 694], we have

$$
h(t) \in \overline{\operatorname{conv}} w-\limsup _{n \rightarrow+\infty}\left\{h_{n}(t)\right\} \subseteq F(t, u(t)) \quad \text { for a.a. } t \in T
$$

(see hypothesis $H(F)_{1}(i i)$ ), so $h \in S_{F(\cdot, u(\cdot))}^{2}$. Hypothesis $H(F)_{1}(i i)$ is a multivalued variant of a condition first used by Hartman [10].

Together with $H(F)_{1}$, we will need the following extra condition on $\partial \varphi$ :

$H_{0}: \quad$ For all $x \in D(\partial \varphi)$ and $g \in \partial \varphi(x)$, we have $(g, x)_{\mathbb{R}^{N}} \geqslant 0$.

Alternatively, instead of $H(F)_{1}, H_{0}$, we can use the following conditions on $F$ :

$H(F)_{1}^{\prime}: F: T \times \mathbb{R}^{N} \longrightarrow P_{k c}\left(\mathbb{R}^{N}\right)$ is a multifunction such that hypotheses $H(F)_{1}^{\prime}(i)$ and (ii) are the same as the corresponding hypotheses $H(F)_{1}(i)$ and $(i i)$ and

(iii) $|F(t, x)| \leqslant k(t)(1+|x|) \mid$ for almost all $t \in T$, all $x \in \mathbb{R}^{N}$, with $k \in L^{2}(T)$.

We know that

$$
\overline{D(\partial \varphi)}=\overline{\operatorname{dom} \varphi}
$$

(see Hu and Papageorgiou [11, p.346]). Let $x_{0} \in \overline{\operatorname{dom} \varphi}, h \in L^{2}\left(T ; \mathbb{R}^{N}\right)$ and $\varepsilon>0$. We consider the following auxiliary Cauchy problem:

$$
\left\{\begin{array}{l}
-u^{\prime}(t) \in A(t, u(t))+\partial \varphi(u(t))+\varepsilon u(t)+h(t) \quad \text { for a.a. } t \in T \\
u(0)=x_{0}
\end{array}\right.
$$

We have the following existence and uniqueness theorem for this problem.

Proposition 3.2 If hypotheses $H(A)$ and $H(\varphi)$ hold, then problem (3.1) admits a unique solution $u_{0} \in W^{1,2}\left((0, b) ; \mathbb{R}^{N}\right) \subseteq C\left(T ; \mathbb{R}^{N}\right)$.

\section{Proof Let}

$$
V(t, x)=A(t, x)+\partial \varphi(x)+\varepsilon x .
$$

For all $t \in T, V(t, \cdot)$ is maximal monotone. Moreover, because of the monotonicity of $x \longmapsto \partial \varphi(x)$ and of $x \longmapsto \varepsilon x, V(t, x)$ satisfies hypothesis $H(A)(i i)$. Therefore, we can apply Theorem 1.2 of Pavel [13] and have a solution $u_{0} \in C\left(T ; \mathbb{R}^{N}\right)$ of problem (3.1). We have $u_{0}(t) \in D(\partial \varphi)$ for all $t \in T$ and recall that on account of hypothesis $H(\varphi), \partial \varphi$ maps bounded sets to bounded sets. Therefore,

$$
\left|\partial \varphi\left(u_{0}(t)\right)\right| \leqslant M \quad \forall t \in T,
$$

for some $M>0$. This fact together with hypothesis $H(A)(\mathrm{iii})$ implies that $u_{0}^{\prime} \in$ $L^{2}\left(T ; \mathbb{R}^{N}\right)$, so $u_{0} \in W^{1,2}\left((0, b) ; \mathbb{R}^{N}\right)$.

Next, we show that this solution is unique. So, suppose that $u_{0}, v_{0} \in W^{1,2}\left((0, b) ; \mathbb{R}^{N}\right)$ are two such solutions. We have

$$
\begin{array}{r}
-u_{0}^{\prime}(t) \in A\left(t, u_{0}(t)\right)+\partial \varphi\left(u_{0}(t)\right)+\varepsilon u_{0}(t)+h(t) \quad \text { for a.a. } t \in T, \\
-v_{0}^{\prime}(t) \in A\left(t, v_{0}(t)\right)+\partial \varphi\left(v_{0}(t)\right)+\varepsilon v_{0}(t)+h(t) \quad \text { for a.a. } t \in T,
\end{array}
$$

with $u_{0}(0)=v_{0}(0)=x_{0}$.

Subtracting (3.3) from Eq. 3.2, we obtain

$$
\begin{aligned}
0 \in & u_{0}^{\prime}(t)-v_{0}^{\prime}(t)+A\left(t, u_{0}(t)\right)-A\left(t, v_{0}(t)\right)+\partial \varphi\left(u_{0}(t)\right)-\partial \varphi\left(v_{0}(t)\right) \\
& +\varepsilon\left(u_{0}(t)-v_{0}(t)\right) \quad \text { for a.a. } t \in T .
\end{aligned}
$$


We take inner product with $u_{0}(t)-v_{0}(t)$. The monotonicity of $A(t, \cdot)$ and $\partial \varphi$ implies that

$$
\frac{1}{2} \frac{d}{d t}\left|u_{0}(t)-v_{0}(t)\right|^{2} \leqslant-\varepsilon\left|u_{0}(t)-v_{0}(t)\right|^{2} \quad \text { for a.a. } t \in T,
$$

so

$$
\left|u_{0}(t)-v_{0}(t)\right|^{2} \leqslant 0
$$

( since $u_{0}(0)=v_{0}(0)=x_{0}$ ), hence $u_{0}=v_{0}$. This proves the uniqueness of the solution of problem (3.1).

We consider the Poincaré map $P: \overline{\operatorname{dom} \varphi} \longrightarrow \overline{\operatorname{dom} \varphi}$ defined by

$$
P\left(x_{0}\right)=u_{0}(b),
$$

with $u_{0} \in W^{1,2}\left((0, b) ; \mathbb{R}^{N}\right)$ being the unique solution of Eq. 3.1 (see Proposition 3.2).

Proposition 3.3 If hypotheses $H(A)$ and $H(\varphi)$ hold, then the Poincaré map $P$ is a contraction.

Proof Let $x_{0}, \widehat{x} \in \overline{\operatorname{dom} \varphi}$ be two distinct initial conditions for problem (3.1) and let $u_{0}, \widehat{u} \in$ $W^{1, p}\left((0, b) ; \mathbb{R}^{N}\right)$ be the corresponding unique solutions of the Cauchy problem. We have

$$
\begin{array}{r}
-u_{0}^{\prime}(t) \in A\left(t, u_{0}(t)\right)+\partial \varphi\left(u_{0}(t)\right)+\varepsilon u_{0}(t)+h(t) \quad \text { for a.a. } t \in T, u_{0}(0)=x_{0} \\
-\widehat{u}^{\prime}(t) \in A(t, \widehat{u}(t))+\partial \varphi(\widehat{u}(t))+\varepsilon \widehat{u}(t)+h(t) \quad \text { for a.a. } t \in T, \widehat{u}(0)=\widehat{x} .
\end{array}
$$

As in the proof of Proposition 3.2, we subtract (3.6) from (3.5) and then take inner product with $u_{0}(t)-\widehat{u}(t)$ to obtain

$$
\frac{1}{2} \frac{d}{d t}\left|u_{0}(t)-\widehat{u}(t)\right|^{2}+\varepsilon\left|u_{0}(t)-\widehat{u}(t)\right| \leqslant 0 \quad \text { for a.a. } t \in T,
$$

so

$$
\frac{d}{d t}\left(e^{2 \varepsilon t}\left|u_{0}(t)-\widehat{u}(t)\right|^{2}\right) \leqslant 0 \quad \text { for a.a. } t \in T
$$

thus

$$
\left|u_{0}(t)-\widehat{u}(t)\right|^{2} \leqslant e^{-2 \varepsilon t}\left|x_{0}-\widehat{x}\right| \quad \text { for a.a. } t \in T .
$$

Let $t=b$. Then,

$$
\mid P\left(x_{0}\right)-P\left(\widehat{x}\left|\leqslant e^{-2 \varepsilon b}\right| x_{0}-\widehat{x} \mid,\right.
$$

so $P$ is a contraction.

Now, for $h \in L^{2}\left(T ; \mathbb{R}^{N}\right)$, we consider the following auxiliary periodic system:

$$
\left\{\begin{array}{l}
-u^{\prime}(t) \in A(t, u(t))+\partial \varphi(u(t))+\varepsilon u(t)+h(t) \quad \text { for a.a. } t \in T=[0, b] \\
u(0)=u(b)
\end{array}\right.
$$

Proposition 3.4 If hypotheses $H(A)$ and $H(\varphi)$ hold, then problem (3.7) admits a unique solution $u_{0} \in W^{1,2}\left((0, b) ; \mathbb{R}^{N}\right) \subseteq C\left(T ; \mathbb{R}^{N}\right)$ and we have

$$
\left|u_{0}(t)\right| \leqslant c_{0}+\int_{0}^{t}|h(s)| d s \quad \forall t \in T,
$$

for some $c_{0}>0$. 
Proof From Proposition 3.3, we know that the Poincaré map $P: \overline{\operatorname{dom} \varphi} \longrightarrow \overline{\operatorname{dom} \varphi}$ is a contraction. So, by the Banach fixed point theorem, there is a unique $u_{0} \in \overline{\operatorname{dom} \varphi}$ such that

$$
P\left(x_{0}\right)=x_{0} \text {. }
$$

The corresponding solution $u_{0} \in W^{1,2}\left((0, b) ; \mathbb{R}^{N}\right)$ of Eq. 3.1 is the unique solution of the periodic system (3.7).

Then on account of hypothesis $H(A)(i i i)$, we can find $g_{0} \in S_{\partial \varphi\left(u_{0}(\cdot)\right)}^{2}$ such that

$$
\left\{\begin{array}{l}
-u_{0}^{\prime}(t) \in A\left(t, u_{0}(t)\right)+g_{0}(t)+\varepsilon u_{0}(t)+h(t) \quad \text { for a.a. } t \in T \\
u_{0}(0)=u_{0}(b) .
\end{array}\right.
$$

We act with $u_{0}(t)$ and recall that $0 \in A(t, 0)$ for all $t \in T$, we obtain

$$
\frac{1}{2} \frac{d}{d t}\left|u_{0}(t)\right|^{2} \leqslant-\varepsilon\left|u_{0}(t)\right|^{2}-\left(g_{0}(t)-v_{0}, u_{0}(t)\right)_{\mathbb{R}^{N}}-\left(v_{0}, u_{0}(t)\right)_{\mathbb{R}^{N}}-\left(h(t), u_{0}(t)\right)_{\mathbb{R}^{N}},
$$

for almost all $t \in T$, all $v_{0} \in \partial \varphi(0)$, so

$$
\frac{1}{2} \frac{d}{d t}\left|u_{0}(t)\right|^{2} \leqslant-\varepsilon\left|u_{0}(t)\right|^{2}+(|\partial \varphi(0)|+|h(t)|)\left|u_{0}(t)\right| \quad \text { for a.a. } t \in T,
$$

thus,

$$
\left|u_{0}(t)\right| \frac{d}{d t}\left|u_{0}(t)\right| \leqslant-\varepsilon\left|u_{0}(t)\right|^{2}+(|\partial \varphi(0)|+|h(t)|)\left|u_{0}(t)\right| \quad \text { for a.a. } t \in T .
$$

It follows that

$$
\left.\frac{d}{d t}\left|u_{0}(t)\right| \leqslant-\varepsilon\left|u_{0}(t)\right|+|\partial \varphi(0)|+|h(t)|\right) \mid \quad \text { for a.a. } t \in T .
$$

Note that

$$
\begin{aligned}
\frac{d}{d t}\left(e^{\varepsilon t}\left|u_{0}(t)\right|\right) & =\varepsilon e^{\varepsilon t}\left|u_{0}(t)\right|+e^{\varepsilon t} \frac{d}{d t}\left|u_{0}(t)\right|=e^{\varepsilon t}\left(\varepsilon\left|u_{0}(t)\right|+\frac{d}{d t}\left|u_{0}(t)\right|\right) \\
& =e^{\varepsilon t}\left(\varepsilon\left|u_{0}(t)\right|-\varepsilon\left|u_{0}(t)\right|+|\partial \varphi(0)|+|h(t)|\right) \\
& =e^{\varepsilon t}(|\partial \varphi(0)|+|h(t)|),
\end{aligned}
$$

thus,

$$
\begin{aligned}
\left|u_{0}(t)\right| & \leqslant e^{-\varepsilon t}\left|u_{0}(0)\right|+e^{-\varepsilon t} \int_{0}^{t} e^{\varepsilon s}(|\partial \varphi(0)|+|h(s)|) d s \\
& \leqslant e^{-\varepsilon t}\left|u_{0}(0)\right|+|\partial \varphi(0)| b+\int_{0}^{t}|h(s)| d s \quad \forall t \in T .
\end{aligned}
$$

In Eq. 3.9, we choose $t=b$. Using the periodic boundary condition, we have

$$
\left(1-e^{-\varepsilon b}\right)\left|u_{0}(0)\right| \leqslant|\partial \varphi(0)| b+\|h\|_{1},
$$

so

$$
\left|u_{0}(0)\right| \leqslant \frac{1}{1-e^{-\varepsilon b}}\left(|\partial \varphi(0)| b+\|h\|_{1}\right) .
$$

We return to Eq. 3.9 and use Eq. 3.10. Then,

$$
\left|u_{0}(t)\right| \leqslant \frac{1}{1-e^{-\varepsilon b}}\left(|\partial \varphi(0)| b+\|h\|_{1}\right)+|\partial \varphi(0)| b+\int_{0}^{t}|h(s)| d s \quad \forall t \in T,
$$

so

$$
\left|u_{0}(t)\right| \leqslant c_{0}+\int_{0}^{t}|h(s)| d s \quad \forall t \in T,
$$

for some $c_{0}>0$. 
Let $M>0$ be as postulated by hypothesis $H(F)_{1}($ iii $)$ and let $p_{M}: \mathbb{R}^{N} \longrightarrow \mathbb{R}^{N}$ be the $M$-radial retraction defined by

$$
p_{M}(x)= \begin{cases}x & \text { if }|x| \leqslant M \\ \frac{M x}{|x|} & \text { if } M<|x| .\end{cases}
$$

We know that $p_{M}$ is nonexpansive (that is, $\left|p_{M}(x)-p_{M}(u)\right| \leqslant|x-u|$ for all $x, u \in \mathbb{R}^{N}$ ). We set

$$
\widehat{F}(t, x)=F\left(t, p_{M}(x)\right) \quad \forall(t, x) \in T \times \mathbb{R}^{N} .
$$

Evidently, $\widehat{F}(t, x)$ satisfies hypotheses $H(F)_{1}(i)$ and $(i i)$ and in addition, we have

$$
|\widehat{F}(t, x)| \leqslant \widehat{a}_{M}(t) \quad \text { for a.a. } t \in T \text {, all } x \in \mathbb{R}^{N} .
$$

In the sequel by $\widehat{S}_{\varepsilon}$, we denote the solution set of the following periodic system

$$
\left\{\begin{array}{l}
-u^{\prime}(t) \in A(t, u(t))+\partial \varphi(u(t))+\varepsilon u(t)+\widehat{F}(t, u(t)) \\
u(0)=u(b) .
\end{array} \text { for a.a. } t \in T=[0, b]\right.
$$

By $S_{\varepsilon}$, we denote the solution set of

$$
\left\{\begin{array}{l}
-u^{\prime}(t) \in A(t, u(t))+\partial \varphi(u(t))+\varepsilon u(t)+F(t, u(t)) \\
u(0)=u(b) .
\end{array} \text { for a.a. } t \in T=[0, b]\right.
$$

In the next proposition, we derive uniform a priori bounds for the elements of these two solution sets.

Proposition 3.5 (a) If hypotheses $H(A), H(\varphi), H(F)_{1}$, and $H_{0}$ hold, then $|u(t)| \leqslant M$ for all $t \in T$, all $u \in \widehat{S}_{\varepsilon}$ (here, $M>0$ is as postulated in hypothesis $H(F)_{1}($ iii $)$ ).

(b) If hypotheses $H(A), H(\varphi)$, and $H(F)_{1}^{\prime}$ hold, then there exists $M>0$ such that $|u(t)| \leqslant M$ for all $t \in T$, all $u \in S_{\varepsilon}$.

Proof (a) Let $u \in \widehat{S}_{\varepsilon} \subseteq W^{1,2}\left((0, b) ; \mathbb{R}^{N}\right)$. We have

$$
\left\{\begin{array}{l}
-u^{\prime}(t) \in A(t, u(t))+\partial \varphi(u(t))+\varepsilon u(t)+h(t) \quad \text { for a.a. } t \in T \\
u(0)=u(b),
\end{array}\right.
$$

with $h \in S_{\widehat{F}(\cdot, u(\cdot))}^{2}$. Suppose that the result is not true. Then, two situations can occur:

(I) $|u(t)|>M$ for all $t \in T$.

(II) There exist $0 \leqslant \eta \leqslant \tau \leqslant b$ such that

$$
|u(\eta)|=M \text { and }|u(t)|>M \quad \forall t \in[\eta, \tau] .
$$
have

Suppose that (I) holds. From Eq. 3.11 for some $a \in S_{A(\cdot, u(\cdot))}^{2}$ and some $g \in S_{\partial \varphi(u(\cdot))}^{2}$, we

$$
-u^{\prime}(t)=a(t)+g(t)+\varepsilon u(t)+h(t) \quad \text { for a.a. } t \in T .
$$

We take inner product with $u(t)$. Then,

$$
\left(u^{\prime}(t), u(t)\right)_{\mathbb{R}^{N}}+(a(t), u(t))_{\mathbb{R}^{N}}+(g(t), u(t))_{\mathbb{R}^{N}}+\varepsilon|u(t)|^{2}+(h(t), u(t))_{\mathbb{R}^{N}}=0
$$

for a.a. $t \in T$. Note that

$$
\left(u^{\prime}(t), u(t)\right)_{\mathbb{R}^{N}}=\frac{1}{2} \frac{d}{d t}|u(t)|^{2} .
$$

Since $a \in S_{A(\cdot, u(\cdot))}^{2}$ and $0 \in A(t, 0)$ for all $t \in T$, we have

$$
(a(t), u(t))_{\mathbb{R}^{N}} \geqslant 0 \quad \text { for a.a. } t \in T .
$$


Hypothesis $H_{0}$ implies that

$$
(g(t), u(t))_{\mathbb{R}^{N}} \geqslant 0 \quad \text { for a.a. } t \in T .
$$

Therefore, finally, we have

$$
\frac{1}{2} \frac{d}{d t}|u(t)|^{2}+\varepsilon|u(t)|^{2}+(h(t), u(t))_{\mathbb{R}^{N}} \leqslant 0 \quad \text { for a.a. } t \in T,
$$

thus,

$$
|u(b)|^{2}+2 \int_{0}^{b}(h(t), u(t))_{\mathbb{R}^{N}} d t<|u(0)|^{2}
$$

(see (I)). Note that

$$
(h(t), u(t))_{\mathbb{R}^{N}}=\frac{|u(t)|}{M}\left(h(t), p_{M}(u(t))\right)_{\mathbb{R}^{N}} \geqslant 0 \quad \text { for a.a. } t \in T
$$

(see hypothesis $H(F)_{1}(i i i)$ ). Using this in Eq. 3.12, we obtain

$$
|u(b)|^{2}<|u(0)|^{2},
$$

a contradiction.

Next, suppose that (II) holds. Then, repeating the above argument on the interval $[\eta, \tau]$, we obtain

$$
M^{2}<|u(\tau)|^{2}<|u(\eta)|^{2}=M^{2},
$$

a contradiction.

So, we conclude that

$$
|u(t)| \leqslant M \quad \forall t \in T, u \in \widehat{S}_{\varepsilon} .
$$

(b) Let $u \in S_{\varepsilon} \subseteq W^{1,2}\left((o, b) ; \mathbb{R}^{N}\right)$. We have

$$
\left\{\begin{array}{l}
-u^{\prime}(t) \in A(t, u(t))+\partial \varphi(u(t))+\varepsilon u(t)+h(t) \quad \text { for a.a. } t \in T \\
u(0)=u(b),
\end{array}\right.
$$

with $h \in S_{F(\cdot, u(\cdot))}^{2}$. Using Eq. 3.8 from Proposition 3.4, we have

$$
|u(t)| \leqslant c_{0}+\int_{0}^{t}|h(s)| d s \leqslant c_{0}+\int_{0}^{t} k(s)(1+|u(s)|) d s \quad \forall t \in T
$$

(see hypothesis $H(F)_{1}^{\prime}(i i i)$ ), so

$$
|u(t)| \leqslant M \quad \forall t \in T, u \in S_{\varepsilon},
$$

for some $M>0$ (using Gronwall's inequality).

On account of Proposition 3.5, we can always replace $F(t, x)$ by $\widehat{F}(t, x)=F\left(t, p_{M}(x)\right)$. Therefore, without any loss of generality, we may assume that

$$
|F(t, x)| \leqslant \vartheta(t) \quad \text { for a.a. } t \in T, \text { all } x \in \mathbb{R}^{N},
$$

with $\vartheta \in L^{2}(T)$.

Proposition 3.4 implies that we can define the solution map $\gamma_{\varepsilon}: L^{2}\left(T ; \mathbb{R}^{N}\right) \longrightarrow$ $C\left(T ; \mathbb{R}^{N}\right)$ which to every $h \in L^{2}\left(T ; \mathbb{R}^{N}\right)$ assigns the unique solution $\gamma_{\varepsilon}(h) \in$ $W^{1,2}\left((0, b) ; \mathbb{R}^{N}\right) \subseteq C\left(T ; \mathbb{R}^{N}\right)$.

Proposition 3.6 If hypotheses $H(A)$ and $H(\varphi)$ hold, then the solution map $\gamma_{\varepsilon}: L^{2}\left(T ; \mathbb{R}^{N}\right) \longrightarrow C\left(T ; \mathbb{R}^{N}\right)$ is completely continuous (that is, if $h_{n} \stackrel{w}{\longrightarrow} h$ in $L^{2}\left(T ; \mathbb{R}^{N}\right)$, then $\gamma_{\varepsilon}\left(h_{n}\right) \longrightarrow \gamma_{\varepsilon}(h)$ in $\left.C\left(T ; \mathbb{R}^{N}\right)\right)$. 
Proof Let $h_{n} \stackrel{w}{\longrightarrow} h$ in $L^{2}\left(T ; \mathbb{R}^{N}\right)$ and set $u_{n}=\gamma_{\varepsilon}\left(u_{n}\right) \in W^{1,2}\left((0, b) ; \mathbb{R}^{N}\right) \subseteq C\left(T ; \mathbb{R}^{N}\right)$ for all $n \in \mathbb{N}$ and $u=\gamma_{\varepsilon}(h) \in W^{1,2}\left((0, b) ; \mathbb{R}^{N}\right) \subseteq C\left(T ; \mathbb{R}^{N}\right)$. We have

$$
\left\{\begin{array}{l}
0 \in u_{n}^{\prime}(t)+A\left(t, u_{n}(t)\right)+\partial \varphi\left(u_{n}(t)\right)+\varepsilon u_{n}(t)+h_{n}(t) \quad \text { for a.a. } t \in T \\
u_{n}(0)=u_{n}(b), n \in \mathbb{N} .
\end{array}\right.
$$

and

$$
\left\{\begin{array}{l}
0 \in u^{\prime}(t)+A(t, u(t))+\partial \varphi(u(t))+\varepsilon u(t)+h(t) \quad \text { for a.a. } t \in T \\
u(0)=u(b) .
\end{array}\right.
$$

As before (see the proof of Proposition 3.2), subtracting (3.15) from Eq. 3.14 and taking inner product with $u_{n}(t)-u(t)$, we obtain

$$
\begin{aligned}
\left|u_{n}(t)-u(t)\right|^{2} \leqslant & \left|u_{n}(0)-u(0)\right|^{2}+\int_{0}^{t}\left(h_{n}(s)-h(s), u(s)-u_{n}(s)\right)_{\mathbb{R}^{N}} d z \\
& -\varepsilon \int_{0}^{t}\left|u_{n}(s)-u(s)\right|^{2} d s \quad \forall t \in T, n \in \mathbb{N} .
\end{aligned}
$$

From Eq. 3.8, we know that

so

$$
\left|u_{n}(t)\right| \leqslant c_{0}+\int_{0}^{t}\left|h_{n}(s)\right| d s \quad \forall t \in T, n \in \mathbb{N}
$$

$$
\left|u_{n}(t)\right| \leqslant c_{1} \quad \forall t \in T, n \in \mathbb{N},
$$

with $c_{1}>0$. Both conditions in hypothesis $H(\varphi)$ imply that $\partial \varphi$ maps bounded sets to bounded sets (see Gasiński-Papageorgiou [9, Problem 3.59]). Therefore,

$$
\left|\partial \varphi\left(u_{n}(t)\right)\right| \leqslant c_{2} \quad \forall t \in T, n \in \mathbb{N},
$$

for some $c_{2}>0$ (see Eq. 3.17). Also, from hypothesis $H(A)($ iii $)$, we have

$$
\left|A\left(t, u_{n}(t)\right)\right| \leqslant k_{0}(t) \quad \text { for a.a. } t \in T \text {, all } n \in \mathbb{N},
$$

with $k_{0} \in L^{2}(T)$. Returning to Eq. 3.14 and using Eqs. 3.17, 3.18, and 3.19, we infer that the sequence $\left\{u_{n}^{\prime}\right\}_{m \geqslant 1} \subseteq L^{2}\left(T ; \mathbb{R}^{N}\right)$ is bounded, so the sequence $\left\{u_{n}\right\}_{n} \geqslant 1 \subseteq W^{1,2}\left((0, b) ; \mathbb{R}^{N}\right)$ is bounded (see Eq. 3.17).

Thus, by passing to a suitable subsequence if necessary, we may assume that

$$
u_{n} \stackrel{w}{\longrightarrow} \widehat{u} \text { in } W^{1,2}\left((0, b) ; \mathbb{R}^{N}\right) \text { and } u_{n} \longrightarrow \widehat{u} \quad \text { in } C\left(T ; \mathbb{R}^{N}\right) .
$$

In Eq. 3.16, we pass to the limit as $n \rightarrow+\infty$ and use Eq. 3.20. Then,

$$
|\widehat{u}(t)-u(t)|^{2} \leqslant|\widehat{u}(0)-u(0)|^{2}-\varepsilon \int_{0}^{t}|\widehat{u}(s)-u(s)|^{2} d s \quad \forall t \in T .
$$

Let $t=b$ and recall that $\widehat{u}(0)=\widehat{u}(b), u(0)=u(b)$. We obtain

$$
0 \leqslant-\varepsilon\|\widehat{u}-u\|_{2}
$$

so $\widehat{u}=u$. Therefore, for the original sequence, we have $u_{n} \longrightarrow u$ in $C\left(T ; \mathbb{R}^{N}\right)$; thus, $\gamma_{\varepsilon}$ is completely continuous.

On account of Eq. 3.13, we introduce the following set:

$$
W=\left\{g \in L^{2}\left(T ; \mathbb{R}^{N}\right):|g(t)| \leqslant \vartheta(t) \text { for a.a. } t \in T\right\} .
$$

Evidently, $W \subseteq L^{2}\left(T ; \mathbb{R}^{N}\right)$ is sequentially weakly compact (Eberlein-Smulian theorem; see Gasiński and Papageorgiou [7, p. 909]). Using Proposition 3.6, we have

$$
K_{\varepsilon}=\gamma_{\varepsilon}(W) \in P_{k}\left(C\left(T ; \mathbb{R}^{N}\right)\right),
$$


so

$$
K_{c}^{\varepsilon}=\overline{\operatorname{conv}} K_{\varepsilon} \in P_{k c}\left(C\left(T ; \mathbb{R}^{N}\right)\right)
$$

(see Gasiński and Papageorgiou [8, p. 852, Theorem 5.86]).

Now, we can have our existence theorem for the "convex problem."

Theorem 3.7 If hypotheses $H(A), H(\varphi)$ and $H(F)_{1}, H_{0}$ or $H(F)_{1}^{\prime}$ hold, then problem (1.1) admits a solution $u_{0} \in W^{1,2}\left((0, b) ; \mathbb{R}^{N}\right)$.

Proof Let $\varepsilon>0$ and consider the following periodic system

$$
\left\{\begin{array}{l}
-u^{\prime}(t) \in A(t, u(t))+\partial \varphi(u(t))+\varepsilon u(t)+F(t, u(t)) \quad \text { for a.a. } t \in T \\
u(0)=u(b) .
\end{array}\right.
$$

Recall that $F(t, x)$ satisfies (3.13). We consider the set $W \subseteq L^{2}\left(T ; \mathbb{R}^{N}\right)$ furnished with the relative weak topology and introduce the multifunction $H_{\varepsilon}: W \longrightarrow P_{k c}(W)$ defined by

$$
H_{\varepsilon}(g)=S_{F\left(\cdot, \gamma_{\varepsilon}(g)(\cdot)\right)}^{2} \quad \forall g \in W .
$$

Let $\left\{\left(g_{n}, h_{n}\right)\right\}_{n} \geqslant 1 \subseteq$ Gr $H_{\varepsilon}$ and assume that

$$
g_{n} \stackrel{w}{\longrightarrow} g \text { and } h_{n} \stackrel{w}{\longrightarrow} h \quad \text { in } L^{2}\left(T ; \mathbb{R}^{N}\right) .
$$

Then Proposition 3.6 and Eq. 3.22 imply that

$$
\gamma_{\varepsilon}\left(g_{n}\right) \longrightarrow \gamma_{\varepsilon}(g) \text { in } C\left(T ; \mathbb{R}^{N}\right) .
$$

Using Proposition 3.9 of $\mathrm{Hu}$ and Papageorgiou [11, p. 694], we have

$$
\begin{aligned}
h(t) & \in \overline{\operatorname{conv}} w-\limsup _{n \rightarrow+\infty} F\left(t, \gamma_{\varepsilon}\left(g_{n}\right)(t)\right) \\
& \subseteq F\left(t, \gamma_{\varepsilon}(g)(t)\right) \quad \text { for a.a. } t \in T
\end{aligned}
$$

(see Eq. 3.23 and hypothesis $\left.H(F)_{1}(i i)\right)$, so $(g, h) \in$ Gr $H_{\varepsilon}$ and thus $H_{\varepsilon}$ has closed graph in $W \times W$.

The set $W$ furnished with the relative weak topology is compact. Also, it is convex. Then, invoking the Kakutani-Ky Fan fixed point theorem (see Papageorgiou and Kyritsi [12, p. 114, Theorem 2.6.7] or Gasiński and Papageorgiou [7, p. 887]), we can find $g_{0}^{\varepsilon} \in W$ such that $g_{0}^{\varepsilon} \in H_{\varepsilon}\left(g_{0}^{\varepsilon}\right)$, so $g_{0}^{\varepsilon} \in S_{F\left(\cdot, \gamma_{\varepsilon}\left(g_{0}^{\varepsilon}\right)(\cdot)\right)}^{2}$.

Now, let $\varepsilon_{n} \longrightarrow 0^{+}$and let

$$
u_{0}^{n}=\gamma_{\varepsilon_{n}}\left(g_{0}^{\varepsilon_{n}}\right) \in W^{1,2}\left((0, b) ; \mathbb{R}^{N}\right) \subseteq C\left(T ; \mathbb{R}^{N}\right) \quad \forall n \in \mathbb{N} .
$$

As before (see the proof of Proposition 3.6), using Eq. 3.8, we have that the sequence $\left\{u_{0}^{n}\right\}_{n \geqslant 1} \subseteq C\left(T ; \mathbb{R}^{N}\right)$ is relatively compact.

Therefore, passing to a subsequence if necessary, we may assume that

$$
u_{0}^{n} \longrightarrow u_{0} \quad \text { in } C\left(T ; \mathbb{R}^{N}\right) \text {. }
$$

From Eqs. 3.21 and 3.24 as before (see the proof of Proposition 3.6), we have that the sequence $\left\{\left(u_{0}^{n}\right)^{\prime}\right\}_{n} \geqslant 1 \subseteq L^{2}\left(T ; \mathbb{R}^{N}\right)$ is bounded. Passing to a next subsequence if necessary, we may assume

$$
\left(u_{0}^{n}\right)^{\prime} \stackrel{w}{\longrightarrow} h \text { in } L^{2}\left(T ; \mathbb{R}^{N}\right) .
$$

For every $n \in \mathbb{N}$ and every $t, s \in T, s<t$, we have

$$
u_{0}^{n}(t)-u_{0}^{n}(s)=\int_{s}^{t}\left(u_{0}^{n}\right)^{\prime}(\tau) d \tau,
$$


so

$$
u_{0}(t)-u_{0}(s)=\int_{s}^{t} h(\tau) d \tau
$$

(see Eqs. 3.24 and 3.25), thus $h=u_{0}^{\prime}$.

Let $\Phi: L^{2}\left(T ; \mathbb{R}^{N}\right) \longrightarrow \overline{\mathbb{R}}=\mathbb{R} \cup\{+\infty\}$ be the integral functional defined by

$$
\Phi(u)= \begin{cases}\int_{0}^{b} \varphi(u(t)) d t & \text { if } \varphi(u(\cdot)) \in L^{1}(T), \\ +\infty & \text { otherwise. }\end{cases}
$$

We know that

$$
\Phi \in \Gamma_{0}\left(L^{2}\left(T ; \mathbb{R}^{N}\right)\right) \text { and partial } \Phi(u)=S_{\partial \varphi(u(\cdot))}^{2} \quad \forall u \in L^{2}\left(T ; \mathbb{R}^{N}\right)
$$

(see Hu and Papageorgiou [11, p. 349]). From Eq. 3.21, we have

$$
-\left(u_{0}^{n}\right)^{\prime}-\varepsilon_{n} u_{0}^{n}-g_{0}^{n} \in \operatorname{Gr}(\mathcal{A}+\partial \Phi)\left(u_{0}^{n}\right) \quad \forall n \in \mathbb{N},
$$

where $\mathcal{A}(u) \in S_{A(\cdot, u(\cdot))}^{2}$ for all $u \in L^{2}\left(T ; \mathbb{R}^{N}\right)$. From Lemma 2.3, we have that $\mathcal{A}$ is maximal monotone. In addition, hypothesis $H(A)($ iii $)$ implies that $D(\mathcal{A})=L^{2}\left(T ; \mathbb{R}^{N}\right)$. Therefore, Theorem 3.2.41 of Gasiński and Papageorgiou [7] implies that $u \longmapsto(\mathcal{A}+\partial \Phi)(u)$ is maximal monotone. Hence, its graph is sequentially closed in $L^{2}\left(T ; \mathbb{R}^{N}\right) \times L^{2}\left(T ; \mathbb{R}^{N}\right)_{w}$. Note that $\left\{g_{0}^{n}\right\}_{n \geqslant 1} \subseteq W$. So, we can say that

$$
g_{0}^{n} \stackrel{w}{\longrightarrow} g \quad \text { in } L^{2}\left(T ; \mathbb{R}^{N}\right)
$$

and $g_{0} \in S_{F\left(\cdot, u_{0}(\cdot)\right)}^{2}$ (see Eq. 3.24 and hypothesis $H(F)_{1}(i i)$ ).

Therefore, if in Eq. 3.26 we pass to the limit as $n \rightarrow+\infty$, then

$$
\left(-u_{0}^{\prime}-g_{0}\right) \in(\mathcal{A}+\partial \Phi)\left(u_{0}\right)
$$

(see Eq. 3.25), so

$$
\left\{\begin{array}{l}
-u_{0}^{\prime}(t) \in A\left(t, u_{0}(t)\right)+\partial \varphi\left(u_{0}(t)\right)+F\left(t, u_{0}(t)\right) \quad \text { for a.a. } t \in T \\
u_{0}(0)=u_{0}(b)
\end{array}\right.
$$

An interesting byproduct of the above proof is the following result concerning the solution set $S_{c} \subseteq C\left(T ; \mathbb{R}^{N}\right)$ of the "convex problem."

Proposition 3.8 If hypotheses $H(A), H(\varphi)$ and $H(F)_{1}, H_{0}$, or $H(F)_{1}^{\prime}$ hold, then $S_{c} \in$ $P_{k}\left(C\left(T ; \mathbb{R}^{N}\right)\right)$.

\section{The Nonconvex Problem}

In this section, we prove an existence theorem for the "nonconvex problem" (that is, the multivalued perturbation has nonconvex values).

In this case, the hypotheses on the multivalued perturbation $F$ are the following:

$H(F)_{2}: F: T \times \mathbb{R}^{N} \longrightarrow P_{f}\left(\mathbb{R}^{N}\right) \backslash \emptyset$ is a multifunction such that

(i) $(t, x) \longrightarrow F(t, x)$ is graph measurable.

(ii) For almost all $t \in T, x \longmapsto F(t, x)$ is lower semicontinuous. 
(iii) There exist $M>0$ and $\widehat{a}_{M} \in L^{2}(T)$ such that

$$
\begin{gathered}
0 \leqslant(h, x)_{\mathbb{R}^{N}} \quad \text { for a.a. } t \in T, \text { all }|x|=M, h \in F(t, x), \\
|F(t, x)| \leqslant \widehat{a}_{M}(t) \quad \text { for a.a. } t \in T,|x| \leqslant M .
\end{gathered}
$$

As before (see Section 3), these hypotheses will be combined with $H_{0}$. Alternatively, instead of the pair $H(F)_{2}, H_{0}$, we can use the following hypotheses on $F$ :

$H(F)_{2}^{\prime}: F: T \times \mathbb{R}^{N} \longrightarrow P_{f}\left(\mathbb{R}^{N}\right)$ is a multifunction such that hypotheses $H(F)_{2}^{\prime}(i)$ and (ii) are the same as the corresponding hypotheses $H(F)_{2}(i)$ and $(i i)$ and

(iii) $|F(t, x)| \leqslant k(t)(1+|x|) \mid$ for almost all $t \in T$, all $x \in \mathbb{R}^{N}$, with $k \in L^{2}(T)$.

Theorem 4.1 If hypotheses $H(A), H(\varphi)$ and $H(F)_{2}, H_{0}$, or $H(F)_{2}^{\prime}$ hold, then problem (1.1) admits a solution $u_{0} \in W^{1,2}\left((0, b) ; \mathbb{R}^{N}\right) \subseteq C\left(T ; \mathbb{R}^{N}\right)$.

Proof The a priori bounds in Proposition 3.5 remain valid and so without any loss of generality, we may assume that

$$
|F(t, x)| \leqslant \vartheta(t) \text { for a.a. } t \in T, \text { all } x \in \mathbb{R}^{N},
$$

with $\vartheta \in L^{2}(T)$ (just replace $F(t, x)$ with $\widehat{F}(t, x)=F\left(t, p_{M}(x)\right)$ ).

Again, we introduce the sets

$$
\begin{aligned}
W & =\left\{g \in L^{2}\left(T ; \mathbb{R}^{N}\right):|g(t)| \leqslant \vartheta(t) \text { for a.a. } t \in T\right\} \\
K_{c}^{\varepsilon} & =\overline{\operatorname{conv}} \gamma_{\varepsilon}(W) \in P_{k c}(C(T ; H)) .
\end{aligned}
$$

We introduce the multifunction $V: C\left(T ; \mathbb{R}^{N}\right) \longrightarrow P_{w k}\left(L^{2}\left(T ; \mathbb{R}^{N}\right)\right)$ defined by

$$
V(u)=S_{F(\cdot, u(\cdot))}^{2} \quad \forall u \in C\left(T ; \mathbb{R}^{N}\right) .
$$

We show that $V$ is a lower semicontinuous multifunction. According to Proposition 2.6 of $\mathrm{Hu}$ and Papageorgiou [11, p. 37], to show the lower semicontinuity of $V$, it suffices to show that, if $u_{n} \longrightarrow u$ in $C(T ; H)$, then

$$
V(u) \subseteq \liminf _{n \rightarrow+\infty} V\left(u_{n}\right) .
$$

So, suppose that

$$
u_{n} \longrightarrow u \text { in } C(T ; H) \text { and } h \in V(u) .
$$

For $n \in \mathbb{N}$, we consider the multifunction $G_{n}: T \longrightarrow P_{k}\left(\mathbb{R}^{N}\right)$ defined by

$$
G_{n}(t)=\left\{v \in F\left(t, u_{n}(t)\right):|h(t)-v| \leqslant d\left(h(t), F\left(t, u_{n}(t)\right)\right)+\frac{1}{n}\right\} .
$$

Hypothesis $H(F)_{2}(i)$ implies that the map $t \longmapsto d\left(h(t), F\left(t, u_{n}(t)\right)\right)$ is measurable. Therefore,

$$
T \times \mathbb{R}^{N} \ni(t, v) \longmapsto e_{n}(t, v)=|h(t)-v|-d\left(h(t), F\left(t, u_{n}(t)\right)\right)
$$

is a Carathéodory function. We know that Carathéodory functions are jointly measurable (see $\mathrm{Hu}$ and Papageorgiou [11, p. 142]). Therefore,

$$
\operatorname{Gr} G_{n}=\left\{(t, v) \in T \times \mathbb{R}^{N}: e_{n}(t, v) \leqslant \frac{1}{n}\right\} \in \mathcal{L}_{T} \otimes \mathcal{B}\left(\mathbb{R}^{N}\right) .
$$

Invoking the Yankov-von Neumann-Aumann selection theorem, we can find a measurable function $h_{n}: T \longrightarrow \mathbb{R}^{N}$ such that

$$
h_{n}(t) \in G_{n}(t) \text { for a.a. } t \in T \text {, all } n \in \mathbb{N} \text {. }
$$


We have

$$
\left|h(t)-h_{n}(t)\right| \leqslant d\left(h(t), F\left(t, u_{n}(t)\right)\right)+\frac{1}{n} \quad \text { for a.a. } t \in T, \text { all } n \in \mathbb{N},
$$

so

$$
\begin{aligned}
\limsup _{n \rightarrow+\infty}\left|h(t)-h_{n}(t)\right| & \leqslant \limsup _{n \rightarrow+\infty} d\left(h(t), F\left(t, u_{n}(t)\right)\right) \\
& \leqslant d\left(h(t), \liminf _{n \rightarrow+\infty} F\left(t, u_{n}(t)\right)\right) \\
& \leqslant d(h(t), F(t, u(t)))=0 \text { for a.a. } t \in T
\end{aligned}
$$

(see $\mathrm{Hu}$ and Papageorgiou [11, p. 672, Proposition 1.47], Eq. 4.2, hypothesis $H(F)_{2}(i i)$ and recall that $h \in V(u))$, thus,

$$
h_{n}(t) \longrightarrow h(t) \text { for a.a. } t \in T .
$$

Also, we have

$$
\left|h(t)-h_{n}(t)\right|^{2} \leqslant 2 \vartheta(t)^{2} \quad \text { for a.a. } t \in T, \text { all } n \in \mathbb{N}
$$

(see Eq. 4.1), so

$$
\left\{\left|\left(h-h_{n}\right)(\cdot)\right|^{2}\right\}_{n \geqslant 1} \subseteq L^{1}(T) \text { is uniformly integrable. }
$$

Then, from Eqs. 4.3 and 4.4 and Vitali's theorem (see, e.g., Gasiński and Papageorgiou [7, p. 901]), we have

$$
h_{n} \longrightarrow h \text { in } L^{2}\left(T ; \mathbb{R}^{N}\right),
$$

with $h_{n} \in V\left(u_{n}\right)$ for all $n \in \mathbb{N}$, thus, $V$ is lower semicontinuous.

Clearly, $V$ has decomposable values. Applying the Bressan-Colombo-Fryszkowski selection theorem (see Bressan and Colombo [2], Fryszkowski [6], and $\mathrm{Hu}$ and Papageorgiou [11, p. 245, Theorem 8.7]), we can find a continuous map $v: C\left(T ; \mathbb{R}^{N}\right) \longrightarrow$ $L^{2}\left(T ; \mathbb{R}^{N}\right)$ such that

$$
v(u) \in V(u) \quad \forall u \in C\left(T ; \mathbb{R}^{N}\right) .
$$

We set $\tau_{\varepsilon}=\gamma_{\varepsilon} \circ v: K_{c}^{\varepsilon} \longrightarrow K_{c}^{\varepsilon}$. Evidently, $\tau_{\varepsilon}$ is continuous (see Proposition 3.6). Since $K_{c}^{\varepsilon} \in P_{k c}\left(C\left(T ; \mathbb{R}^{N}\right)\right)$, we can apply the Schauder fixed point theorem and produce $u_{0}^{\varepsilon} \in K_{c}^{\varepsilon}$ such that

$$
u_{0}^{\varepsilon}=\tau_{\varepsilon}\left(u_{0}^{\varepsilon}\right),
$$

so $\left.u_{0}^{\varepsilon} \in W^{1,2}\left((0, b) ; \mathbb{R}^{N}\right)\right)$ is a solution of problem $(3.21)$.

Now, consider a sequence $\varepsilon_{n} \rightarrow 0^{+}$and let $u_{n}=u_{0}^{\varepsilon_{n}}$ for $n \in \mathbb{N}$. From Eqs. 3.8 and 4.1, we see that there exists $c_{\varepsilon}>0$ such that

$$
\left|u_{n}(t)\right| \leqslant c_{3} \quad \forall t \in T, n \in \mathbb{N} .
$$

This implies that Eqs. 3.18 and 3.19 are valid and hence the sequence $\left\{u_{n}^{\prime}\right\}_{n} \geqslant 1 \subseteq$ $L^{2}\left(T ; \mathbb{R}^{N}\right)$ is bounded.

For all $t, s \in T, s \leqslant t$, by the Cauchy-Schwarz inequality, we have

$$
\left|u_{n}(t)-u_{n}(s)\right| \leqslant \int_{s}^{t}\left|u_{n}^{\prime}(t)\right| d \tau \leqslant(t-s)^{\frac{1}{2}}\left\|u_{n}^{\prime}\right\|_{2} \leqslant c_{4}(t-s)^{\frac{1}{2}} \quad \forall n \geqslant 1,
$$

for some $c_{4}>0$, so, the sequence $\left\{u_{n}\right\}_{n} \geqslant 1$ is equicontinuous.

The Arzela-Ascoli theorem implies that the sequence $\left\{u_{0}^{\varepsilon_{n}}\right\}_{n} \geqslant 1 \subseteq C\left(T ; \mathbb{R}^{N}\right)$ is relatively compact.

So, passing to a subsequence if necessary, we may assume that

$$
u_{0}^{\varepsilon_{n}} \longrightarrow u_{0} \quad \text { in } C\left(T ; \mathbb{R}^{N}\right) \text {. }
$$


Exploiting the continuity of $V$ and reasoning as in the last part of the proof of Theorem 3.7, in the limit as $n \rightarrow+\infty$, we obtain

$$
\left\{\begin{array}{l}
-u_{0}^{\prime}(t) \in A\left(t, u_{0}(t)\right)+\partial \varphi\left(u_{0}(t)\right)+F\left(t, u_{0}(t)\right) \quad \text { for a.a. } t \in T \\
u_{0}(0)=u_{0}(b)
\end{array}\right.
$$

so $u_{0} \in W^{1,2}\left((0, b) ; \mathbb{R}^{N}\right) \subseteq C\left(T ; \mathbb{R}^{N}\right)$ is a solution of Eq. 1.1 .

\section{Extremal Trajectories}

In this section, we deal with the following version of problem (1.1):

$$
\left\{\begin{array}{l}
-u^{\prime}(t) \in A(t, u(t))+\partial \varphi(u(t))+\operatorname{ext} F(t, u(t)) \quad \text { for a.a. } t \in T \\
u(0)=u(b) .
\end{array}\right.
$$

Here, by ext $F(t, x)$, we denote the set of extreme points of $F(t, x)$. We know that even if $F(t, \cdot)$ has strong continuity properties, the multifunction $x \longmapsto$ ext $F(t, x)$ need not have any (see Hu and Papageorgiou [11, Section 2.4]). So, the existence of solutions for problem (5.1) cannot be deduced from Theorems 3.7 and 4.1 and a different approach is needed.

We need to strengthen the conditions on the multivalued perturbation $F(t, x)$. The new hypotheses are the following:

$H(F)_{3}: F: T \times \mathbb{R}^{N} \longrightarrow P_{k c}\left(\mathbb{R}^{N}\right)$ is a multifunction such that

(i) For all $x \in \mathbb{R}^{N}, t \longrightarrow F(t, x)$ is graph measurable.

(ii) For almost all $t \in T, x \longmapsto F(t, x)$ is $h$-continuous.

(iii) There exist $M>0$ and $\widehat{a}_{M} \in L^{2}(T)$ such that

$$
\begin{gathered}
0 \leqslant(h, x)_{\mathbb{R}^{N}} \quad \text { for a.a. } t \in T, \text { all }|x|=M, h \in F(t, x), \\
|F(t, x)| \leqslant \widehat{a}_{M}(t) \quad \text { for a.a. } t \in T,|x| \leqslant M .
\end{gathered}
$$

As before, $H(F)_{3}$ will be combined with $H_{0}$. Alternatively, we can replace the pair $H(F)_{3}, H_{0}$ with the following hypotheses:

$H(F)_{3}^{\prime}: F: T \times \mathbb{R}^{N} \longrightarrow P_{k c}\left(\mathbb{R}^{N}\right) \backslash \emptyset$ is a multifunction such that hypotheses $H(F)_{3}^{\prime}(i)$ and $(\mathrm{ii})$ are the same as the corresponding hypotheses $H(F)_{3}(i)$ and $(i i)$ and

(iii) $|F(t, x)| \leqslant k(t)(1+|x|) \mid$ for almost all $t \in T$, all $x \in \mathbb{R}^{N}$, with $k \in L^{2}(T)$.

Theorem 5.1 If hypotheses $H(A), H(\varphi)$ and $H(F)_{3}, H_{0}$, or $H(F)_{3}^{\prime}$ hold, then problem (5.1) admits a solution $u_{0} \in W^{1,2}\left((0, b) ; \mathbb{R}^{N}\right) \subseteq C\left(T ; \mathbb{R}^{N}\right)$.

Proof Given $\varepsilon \in(0,1]$, first we consider the following regularization of Eq. 5.1:

$$
\left\{\begin{array}{l}
-u^{\prime}(t) \in A(t, u(t))+\partial \varphi(u(t))+\varepsilon u(t)+\operatorname{ext} F(t, u(t)) \text { for a.a. } t \in T \\
u(0)=u(b)
\end{array}\right.
$$

The a priori bound from Proposition 3.5 remains valid. So, without any loss of generality we assume that

$$
|F(t, x)| \leqslant \vartheta(t) \text { for a.a. } t \in T, \text { all } x \in \mathbb{R}^{N},
$$

with $\vartheta \in L^{2}(T)$. As before

$$
W=\left\{g \in L^{2}\left(T ; \mathbb{R}^{N}\right):|g(t)| \leqslant \vartheta(t) \text { for a.a. } t \in T\right\},
$$


while from the proof of Proposition 3.4 (see Eq. 3.10), we see that we can find $\widehat{K}_{c} \in$ $P_{k c}\left(C\left(T ; \mathbb{R}^{N}\right)\right)$ such that $\overline{\operatorname{conv}} \gamma_{\varepsilon}(W) \subseteq \widehat{K}_{c}$ for all $\varepsilon \in(0,1]$.

Applying Theorem 8.31 of Hu and Papageorgiou [11, p. 260], we can find a continuous $\operatorname{map} \tau: \widehat{K}_{c} \longrightarrow L_{w}^{1}\left(T ; \mathbb{R}^{N}\right)$ such that

$$
\tau(u) \in \operatorname{ext} S_{F(\cdot, u(\cdot))}^{2}=S_{\text {ext } F(\cdot, u(\cdot))}^{2} \forall u \in \widehat{K}_{c}
$$

(see $\mathrm{Hu}$ and Papageorgiou [11, p. 191, Theorem 4.5]). Then, we consider the map $\sigma_{\varepsilon}=$ $\gamma_{\varepsilon} \circ \tau: \widehat{K}_{c} \longrightarrow \widehat{K}_{c}$. We claim that $\sigma_{\varepsilon}$ is continuous. So, let $\left\{u_{n}\right\}_{n \geqslant 1} \subseteq \widehat{K}_{c}$ and assume that

$$
u_{n} \longrightarrow u \in \widehat{K}_{c} \quad \text { in } C\left(T ; \mathbb{R}^{N}\right),
$$

so

$$
\tau\left(u_{n}\right) \longrightarrow \tau(u) \quad \text { in } L_{w}^{1}\left(T ; \mathbb{R}^{N}\right) .
$$

Because of Eq. 5.3, we can apply Lemma 2.8 of Hu and Papageorgiou [11, p. 24] and have

$$
\tau\left(u_{n}\right) \stackrel{w}{\longrightarrow} \tau(u) \quad \text { in } L^{2}\left(T ; \mathbb{R}^{N}\right),
$$

thus,

$$
\gamma_{\varepsilon}\left(\tau\left(u_{n}\right)\right) \longrightarrow \gamma_{\varepsilon}(\tau(u)) \quad \text { in } C\left(T ; \mathbb{R}^{N}\right),
$$

thus, $\sigma_{\varepsilon}$ is continuous.

Since $\sigma_{\varepsilon}: \widehat{K}_{c} \longrightarrow \widehat{K}_{c}$ and $\widehat{K}_{c} \in P_{k c}\left(C\left(T ; \mathbb{R}^{N}\right)\right)$, the Schauder fixed point theorem gives $u_{0}^{\varepsilon} \in \widehat{K}_{c}$ such that $u_{0}^{\varepsilon}=\sigma_{\varepsilon}\left(u_{0}^{\varepsilon}\right)$.

Let $\varepsilon_{n} \in(0,1]$ for $n \in \mathbb{N}$ and assume that $\varepsilon_{n} \rightarrow 0^{+}$. We set $u_{0}^{n}=u_{0}^{\varepsilon_{n}}$ for $n \in \mathbb{N}$. From the proof of Theorem 4.1, we have that the sequence $\left\{u_{0}^{n}\right\}_{n} \geqslant 1 \subseteq C\left(T ; \mathbb{R}^{N}\right)$ is relatively compact. So, passing to a subsequence if necessary, we may assume that

$$
u_{0}^{n} \longrightarrow u_{0} \quad \text { in } C\left(T ; \mathbb{R}^{N}\right) \text {. }
$$

For every $n \in \mathbb{N}$, we have

$$
\left\{\begin{array}{l}
-\left(u_{0}^{n}\right)^{\prime}(t) \in A\left(t, u_{0}^{n}(t)\right)+\partial \varphi\left(u_{0}^{n}(t)\right)+\varepsilon_{n} u_{0}^{n}(t)+\tau\left(u_{0}^{n}\right)(t) \quad \text { for a.a. } t \in T \\
u_{0}^{n}(0)=u_{0}^{n}(b) .
\end{array}\right.
$$

Since $\tau\left(u_{0}^{n}\right) \stackrel{w}{\longrightarrow} \tau\left(u_{0}\right)$ in $L^{2}\left(T ; \mathbb{R}^{N}\right)$ (see Lemma 2.8 of Hu and Papageorgiou [11, p. 24]) as in the proof of Theorem 3.7, in the limit as $n \rightarrow+\infty$, we obtain

$$
\left\{\begin{array}{l}
-u_{0}^{\prime}(t) \in A\left(t, u_{0}(t)\right)+\partial \varphi\left(u_{0}(t)\right)+\operatorname{ext} F\left(t, u_{0}(t)\right) \text { for a.a. } t \in T \\
u_{0}(0)=u_{0}(b)
\end{array}\right.
$$

(see Eq. 5.4). Therefore, $u_{0} \in W^{1,2}\left((0, b) ; \mathbb{R}^{N}\right) \subseteq C\left(T ; \mathbb{R}^{N}\right)$ is a solution of Eq. 5.1.

\section{Strong Relaxation}

In this section, we show that every solution of the convex problem can be obtained as the limit in the $C\left(T ; \mathbb{R}^{N}\right)$-norm of certain extremal trajectories. Such a result is known as "strong relaxation." The result is important in many applications. In the context of control systems, it says that we can approximate any state of the system by states which are generated using "bang-bang controls." This way, we can economize in the use of controls. In the context of game theory, the selection of ext $F(\cdot, u(\cdot))$ are known as "pure strategies" and the strong relaxation theorem implies that any state can be approximated by ones generated using only pure strategies. 
To prove such a result, we need to strengthen further the conditions on $F$. The new hypotheses are the following:

$H(F)_{4}: F: T \times \mathbb{R}^{N} \longrightarrow P_{k c}\left(\mathbb{R}^{N}\right)$ is a multifunction such that

(i) For all $x \in \mathbb{R}^{N}, t \longrightarrow F(t, x)$ is graph measurable.

(ii) For every $r>0$, there exists $\eta_{r} \in L^{1}(T)$ such that

$$
h(F(t, x), F(t, y)) \leqslant \eta_{r}(t)|x-y|
$$

for almost all $t \in T$, all $x, y \in \mathbb{R}^{N}$ with $|x|,|y| \leqslant r$.

(iii) Tthere exist $M>0$ and $\widehat{a}_{M} \in L^{2}(T)$ such that

$$
\begin{gathered}
0 \leqslant(h, x)_{\mathbb{R}^{N}} \quad \text { for a.a. } t \in T, \text { all }|x|=M, h \in F(t, x), \\
|F(t, x)| \leqslant \widehat{a}_{M}(t) \quad \text { for a.a. } t \in T,|x| \leqslant M .
\end{gathered}
$$

These hypotheses go together with $H_{0}$. Alternatively, the pair $H(F)_{4}, H_{0}$ can be replaced by the following hypotheses:

$H(F)_{4}^{\prime}: F: T \times \mathbb{R}^{N} \longrightarrow P_{k c}\left(\mathbb{R}^{N}\right) \backslash \emptyset$ is a multifunction such that hypotheses $H(F)_{4}^{\prime}(i)$ and (ii) are the same as the corresponding hypotheses $H(F)_{4}(i)$ and $(i i)$ and

(iii) $|F(t, x)| \leqslant k(t)(1+|x|) \mid$ for almost all $t \in T$, all $x \in \mathbb{R}^{N}$, with $k \in L^{2}(T)$.

In what follows by $S_{c}$, we denote the solution set of the convex problem (that is, in Eq. 1.1, $F(\cdot, \cdot)$ has values in $\left.P_{k c}\left(\mathbb{R}^{N}\right)\right)$. From Proposition 3.8, we know that $S_{c} \in$ $P_{k}\left(C\left(T ; \mathbb{R}^{N}\right)\right)$.

Let $x_{0} \in \mathbb{R}^{N}$ and consider the following Cauchy problem:

$$
\left\{\begin{array}{l}
-u^{\prime}(t) \in A(t, u(t))+\partial \varphi(u(t))+\operatorname{ext} F(t, u(t)) \quad \text { for a.a. } t \in T \\
u(0)=x_{0} .
\end{array}\right.
$$

Let $S_{e}\left(x_{0}\right) \subseteq W^{1, p}\left((0, b) ; \mathbb{R}^{N}\right)$ be the solution set of Eq. 6.1. A simplified version of the proof of Theorem 5.1 shows that $S_{e}\left(x_{0}\right) \neq \emptyset$. Then, our strong relaxation result reads as follows.

Theorem 6.1 If hypotheses $H(A), H(\varphi)$ and $H(F)_{4}, H_{0}$, or $H(F)_{4}^{\prime}$ hold and $u \in S_{c}$, then there exists a sequence $\left\{u_{n}\right\}_{n \geqslant 1} \subseteq S_{e}(u(0))$ such that $u_{n} \longrightarrow u$ in $C\left(T ; \mathbb{R}^{N}\right)$.

Proof From the previous work, we know that without any loss of generality, we may assume that

$$
|F(t, x)| \leqslant \vartheta(t) \quad \text { for a.a. } t \in T, \text { all } x \in \mathbb{R}^{N},
$$

with $\vartheta \in L^{2}(T)$.

We know that $S_{c} \in P_{k}\left(C\left(T ; \mathbb{R}^{N}\right)\right)$. Similarly, ${\overline{S_{e}(u(0))}}^{C(T ; H)} \in P_{k}\left(C\left(T ; \mathbb{R}^{N}\right)\right)$. We set

$$
K^{*}=\overline{\operatorname{conv}}\left(S_{c} \cup S_{e}(u(0))\right) \in P_{k c}\left(C\left(T ; \mathbb{R}^{N}\right)\right) .
$$

Since $u \in S_{c}$, we can find $h \in S_{F(\cdot, u(\cdot))}^{2}$ such that

$$
\left\{\begin{array}{l}
-u^{\prime}(t) \in A(t, u(t))+\partial \varphi(u(t))+h(t) \quad \text { for a.a. } t \in T \\
u(0)=u(b)
\end{array}\right.
$$

Given $v \in K^{*}$ and $\varepsilon>0$, we introduce the multifunction $L_{v, \varepsilon}: T \longrightarrow 2^{\mathbb{R}^{N}} \backslash \emptyset$ defined by

$$
L_{v, \varepsilon}(t)=\left\{y \in F(t, v(t)):|h(t)-y|<\frac{1}{2 M_{0} b}+d(h(t), F(t, v(t)))\right\},
$$


with $h \in L^{2}\left(T ; \mathbb{R}^{N}\right)$ as in Eq. 6.3 and $M_{0}=\left|K^{*}\right|$. Hypotheses $H(F)_{4}(i)$ and $(i i)$ imply that $(t, x) \longmapsto F(t, x)$ is graph measurable. So, $t \longmapsto F(t, v(t))$ is measurable. Therefore, $(t, y) \longmapsto \xi(t, y)=|h(t)-y|-d(h(t), F(t, v(v)))$ is a Carathéodory function. We have

$$
\operatorname{Gr} L_{v, \varepsilon}=\left\{(t, y) \in T \times \mathbb{R}^{N}: \xi(t, y)<\frac{\varepsilon}{2 M_{0} b}\right\} \cap \operatorname{Gr} F(\cdot, v(\cdot)),
$$

so $\operatorname{Gr} L_{v, \varepsilon} \in \mathcal{L}_{T} \otimes \mathcal{B}\left(\mathbb{R}^{N}\right)$. Invoking the Yankov-von Neumann-Aumann selection theorem, we can find a measurable function $l_{v, \varepsilon}: T \longrightarrow \mathbb{R}^{N}$ such that

$$
l_{v, \varepsilon}(t) \in L_{v, \varepsilon}(t) \quad \text { for a.a. } t \in T \text {, }
$$

hence,

$$
l_{v, \varepsilon} \in L^{2}\left(T ; \mathbb{R}^{N}\right)
$$

(see Eq. 6.2). Then, we introduce the multifunction $H_{\varepsilon}: K^{*} \longrightarrow 2^{L^{2}\left(T ; \mathbb{R}^{N}\right)}$ defined by

$$
H_{\varepsilon}(v)=S_{L_{v, \varepsilon}}^{2}
$$

From Eq. 6.4, we see that

$$
H_{\varepsilon}(v) \neq \emptyset \quad \forall v \in K^{*}
$$

Also Lemma 8.3 of $\mathrm{Hu}$ and Papageorgiou [11, p. 239] implies that $v \longmapsto H_{\varepsilon}(v)$ is lower semicontinuous and so $v \longmapsto \overline{H_{\varepsilon}(v)}$ is lower semicontinuous (see $\mathrm{Hu}$ and Papageorgiou [11, p. 50]). Clearly, $\overline{H_{\varepsilon}(\cdot)}$ has decomposable values. Hence, the selection theorem of Bressan-Colombo-Fryszkowski (see Bressan and Colombo [2] and Fryszkowski [6]) gives a continuous map $\tau_{\varepsilon}: K^{*} \longrightarrow L^{2}\left(T ; \mathbb{R}^{N}\right)$ satisfying

$$
\tau_{\varepsilon}(v) \in \overline{H_{\varepsilon}(v)} \quad \forall v \in K^{*} .
$$

Moreover, invoking Theorem 8.31 of $\mathrm{Hu}$ and Papageorgiou [11, p. 260], we can find a continuous map $r_{\varepsilon}: K^{*} \longrightarrow L_{w}^{1}\left(T ; \mathbb{R}^{N}\right)$ such that

$$
r_{\varepsilon}(v) \in \operatorname{ext} S_{F(\cdot, v(\cdot))}^{2}=S_{\operatorname{ext} F(\cdot, v(\cdot))}^{2} \text { and }\left\|r_{\varepsilon}(v)-\tau_{\varepsilon}(v)\right\|_{w} \leqslant \varepsilon \quad \forall v \in K^{*} .
$$

Now, let $\varepsilon_{n}=\frac{1}{n}, \tau_{n}=\tau_{\varepsilon_{n}}$, and $r_{n}=r_{\varepsilon_{n}}$ for all $n \in \mathbb{N}$ and $x_{0}=u(0)=u(b)$. We consider the following Cauchy problem:

$$
\left\{\begin{array}{l}
-u^{\prime}(t) \in A(t, u(t))+\partial \varphi(u(t))+r_{n}(u)(t) \quad \text { for a.a. } t \in T \\
u(0)=x_{0}
\end{array}\right.
$$

Let $u_{n} \in W^{1,2}\left((0, b) ; \mathbb{R}^{N}\right)$ be a solution of Eq. 6.7. Evidently, $\left\{u_{n}\right\}_{n \geqslant 1} \subseteq S_{e}\left(x_{0}\right)$ (see Eq. 6.6). Then, we have that the sequence $\left\{u_{n}\right\}_{n} \geqslant 1 \subseteq W^{1,2}\left((0, b) ; \mathbb{R}^{N}\right)$ is bounded, so the sequence $\left\{u_{n}\right\}_{n \geqslant 1} \subseteq C\left(T ; \mathbb{R}^{N}\right)$ is relatively compact. Passing to a subsequence if necessary, we may assume that

$$
u_{n} \stackrel{w}{\longrightarrow} \widehat{u} \quad \text { in } W^{1,2}\left((0, b) ; \mathbb{R}^{N}\right) \text { and } u_{n} \longrightarrow \widehat{u} \quad \text { in } C\left(T ; \mathbb{R}^{N}\right) .
$$


Exploiting the monotonicity of $A(t, \cdot)$ and of $\partial \varphi$ and recalling that $u_{n}(0)=x_{0}=u(0)$ for all $n \in \mathbb{N}$ (see Eq. 6.7), we obtain

$$
\begin{aligned}
\left|u_{n}(t)-u(t)\right|^{2} \leqslant & \int_{0}^{t}\left(r_{n}\left(u_{n}\right)(s)-h(s), u(s)-u_{n}(s)\right)_{\mathbb{R}^{N}} d s \\
= & \int_{0}^{t}\left(r_{n}\left(u_{n}\right)(s)-\tau_{n}\left(u_{n}\right)(s), u(s)-u_{n}(s)\right)_{\mathbb{R}^{N}} d s \\
& +\int_{0}^{t}\left(\tau_{n}\left(u_{n}\right)(s)-h(s), u(s)-u_{n}(s)\right)_{\mathbb{R}^{N}} d s \\
\leqslant & \int_{0}^{t}\left(r_{n}\left(u_{n}\right)(s)-\tau_{n}\left(u_{n}\right)(s), u(s)-u_{n}(s)\right)_{\mathbb{R}^{N}} d s \\
& +\int_{0}^{t}\left|\tau_{n}\left(u_{n}\right)(s)-h(s)\right|\left|u(s)-u_{n}(s)\right| d s \\
\leqslant & \int_{0}^{t}\left(r_{n}\left(u_{n}\right)(s)-\tau_{n}\left(u_{n}\right)(s), u(s)-u_{n}(s)\right)_{\mathbb{R}^{N}} d s \\
& +\int_{0}^{t}\left(\frac{1}{2 M_{0} b n}+d\left(h(s), f\left(s, u_{n}(s)\right)\right)\left|u_{n}(s)-u(s)\right| d s\right. \\
\leqslant & \int_{0}^{t}\left(r_{n}\left(u_{n}\right)(s)-\tau_{n}\left(u_{n}\right)(s), u(s)-u_{n}(s)\right)_{\mathbb{R}^{N}} d s \\
& +\frac{1}{n}+\int_{0}^{t} h\left(F(s, u(s)), F\left(s, u_{n}(s)\right)\right)\left|u_{n}(s)-u(s)\right| d s \\
\leqslant & \int_{0}^{t}\left(r_{n}\left(u_{n}\right)(s)-\tau_{n}\left(u_{n}\right)(s), u(s)-u_{n}(s)\right)_{\mathbb{R}^{N}} d s \\
& +\int_{0}^{t} \eta_{M_{0}}(s)\left|u(s)-u_{n}(s)\right|^{2} d s+\frac{1}{n}
\end{aligned}
$$

(see Eq. 6.5, hypotheses $H(F)_{4}(i i)$ and $H(F)_{4}^{\prime}(i i)$ ). From Eq. 6.6, we have

$$
r_{n}\left(u_{n}\right)-\tau_{n}\left(u_{n}\right) \longrightarrow 0 \quad \text { in } L_{w}^{1}\left(T ; \mathbb{R}^{N}\right) .
$$

Then, Lemma 2.8 of $\mathrm{Hu}$ and Papageorgiou [11, p. 24] implies that

$$
r_{n}\left(u_{n}\right)-\tau_{n}\left(u_{n}\right) \stackrel{w}{\longrightarrow} 0 \quad \text { in } L^{2}\left(T ; \mathbb{R}^{N}\right) .
$$

We return to Eq. 6.9, pass to the limit as $n \rightarrow+\infty$ and use Eqs. 6.8 and 6.20. Then,

$$
|\widehat{u}(t)-u(t)|^{2} \leqslant \int_{0}^{t} \eta_{M_{0}}(s)|\widehat{u}(s)-u(s)|^{2} d s \quad \forall t \in T,
$$

so

$$
|\widehat{u}(t)-u(t)|^{2} \leqslant 0 \quad \forall t \in T
$$

(by Gronwall's inequality). It follows that $\widehat{u}=u$. Therefore, $u=\lim _{n \rightarrow+\infty} u_{n}$ in $C\left(T ; \mathbb{R}^{N}\right)$ and $u_{n} \in S_{e}(u(0))$ for all $n \in \mathbb{N}$.

Let $S_{e} \subseteq W^{1,2}\left((0, b) ; \mathbb{R}^{N}\right) \subseteq C\left(T ; \mathbb{R}^{N}\right)$ be the solution set of problem (5.1). From Theorem 3.7, we know that $S_{e} \neq \emptyset$. If we strengthen the conditions on $A(t, \cdot)$, we can show that the set $S_{e}$ is dense in $S_{c}$ for the $C\left(T ; \mathbb{R}^{N}\right)$-norm topology.

The stronger conditions on $A$ are the following: 
$H(A)^{\prime}: A: T \times \mathbb{R}^{N} \longrightarrow 2^{\mathbb{R}^{N}} \backslash \emptyset$ is a multifunction such that $0 \in A(t, 0)$ for all $t \in T$, hypotheses $H(A)^{\prime}(i)$, (ii), and (iii) are the same as the corresponding hypotheses $H(A)(i),(i i)$, and $(i i i)$ and

(iv) For every $M>0$, there exists $c>0$ such that

$$
c_{M}|x-y|^{2} \leqslant(A(t, x)-A(t, y), x-y)_{\mathbb{R}^{N}}
$$

for all $t \in T$, all $x, y \in \mathbb{R}^{N}$ with $|x|,|y| \leqslant M$.

Example 6.2 Let $\eta \in C\left(T ; \mathbb{R}^{N}\right), \eta \geqslant \widehat{c}>0, A_{0}: \mathbb{R}^{N} \longrightarrow 2^{\mathbb{R}^{N}} \backslash \emptyset$ is a maximal monotone map with $0 \in A_{0}(0)$ and set

$$
A(t, x)=\eta(t)|x|^{p-2} x+A_{0}(x),
$$

with $1<p \leqslant 2$. This map satisfies hypotheses $H(A)^{\prime}$.

Theorem 6.3 If hypotheses $H(A)^{\prime}, H(\varphi)$ and $H(F)_{4}, H_{0}$, or $H(F)_{4}^{\prime}$ hold with

$$
\eta_{M}(t)<c_{M} \text { for a.a. } t \in T \text {, all } M>0,
$$

then, $\bar{S}_{e}^{C\left(T ; \mathbb{R}^{N}\right)}=S_{c}$.

Proof We follow the proof of Theorem 6.1, using this time instead of problem (6.7), the periodic problem (that is, the boundary condition in Eq. 6.7 will be $u(0)=u(b)$ ). So, $\left\{u_{n}\right\}_{n \geqslant 1} \subseteq S_{e}$. Using the periodic boundary condition and hypothesis $H(A)^{\prime}(i v)$, we have (see Eq. 6.9 with $t=b$ )

$$
\begin{aligned}
0 \leqslant & -c_{M_{0}}\left\|u_{n}-u\right\|_{2}^{2}+\int_{0}^{b}\left(r_{n}\left(u_{n}\right)(s)-\tau_{n}\left(u_{n}\right)(s), u(s)-u_{n}(s)\right)_{\mathbb{R}^{N}} d s \\
& +\int_{0}^{b} \eta_{M_{0}}(s)\left|u_{n}(s)-u(s)\right|^{2} d s+\frac{1}{n} \quad \forall n \in \mathbb{N} .
\end{aligned}
$$

Passing to the limit as $n \rightarrow+\infty$, we obtain

$$
0 \leqslant \int_{0}^{b}\left(\eta_{M_{0}}(s)-c_{M_{0}}\right)|\widehat{u}(s)-u(s)|^{2} d s
$$

(see Eqs. 6.20 and 6.8), so $\widehat{u}=u$ (see hypothesis $H(A)^{\prime}(i v)$ ).

Therefore, $u=\lim _{n \rightarrow+\infty} u_{n}$ in $C\left(T ; \mathbb{R}^{N}\right)$ with $u_{n} \in S_{e}$ for all $n \in \mathbb{N}$ (see Eq. 6.8).

\section{An Example}

In this section, we illustrate our results by examining the following periodic control system with a priori feedback:

$$
\left\{\begin{array}{l}
-u^{\prime}(t) \in \xi(t)|u(t)|^{p-2} u(t)+\partial|u(t)|+f(t, u(t))+L(v(t)) \\
u(0)=u(b), v(t) \in C(t, u(t)) \text { for a.a. } t \in T .
\end{array}\right.
$$

In this problem,

$$
\xi \in C(T ; \mathbb{R}), \xi \geqslant 0, \xi \not \equiv 0 \text {, and } 1<p<+\infty .
$$


Also, $\mathbb{R}^{N} \ni x \longmapsto \partial|x|$ denotes the subdifferential in the sense of convex analysis. We know that

$$
\partial|x|= \begin{cases}\frac{x}{|x|} & \text { if } x \neq 0, \\ \bar{B}_{1} & \text { if } x=0,\end{cases}
$$

with $\bar{B}_{1}=\left\{x \in \mathbb{R}^{N}:|x| \leqslant 1\right\}$ (see Gasiński and Papageorgiou [7]). The function $f: T \times \mathbb{R}^{N} \longrightarrow \mathbb{R}^{N}$ is measurable in $t \in T$ and locally $L^{1}(T)$-Lipschitz in $x \in \mathbb{R}$, that is, for every $M^{\prime}>0$, there exists $\eta_{M^{\prime}}^{1} \in L^{1}(T)$ such that

$$
|f(t, x)-f(t, y)| \leqslant \eta_{M^{\prime}}^{1}(t)|x-y| \text { for a.a. } t \in T \text {, all } x, y \in \mathbb{R}^{N},|x|,|y| \leqslant M^{\prime} .
$$

The function $v: T \longrightarrow \mathbb{R}^{m}$ is the control function and $C: T \times \mathbb{R}^{N} \longrightarrow P_{k c}\left(\mathbb{R}^{m}\right)$ is the control constraint multifunction. The dependence of $C$ on $x \in \mathbb{R}^{N}$ implies that there is a priori feedback in the system. We assume that

- For all $x \in \mathbb{R}^{N}, t \longmapsto C(t, x)$ is graph measurable.

- For almost all $t \in T, x \longmapsto C(t, x)$ is locally $L^{1}(T) h$-Lipschitz, that is, for every $M^{\prime}>0$, there exists $\eta_{M^{\prime}}^{2} \in L^{1}(T)$ such that

$$
h(C(t, x), C(t, y)) \leqslant \eta_{M^{\prime}}^{2}(t)|x-y| \quad \text { for a.a. } t \in T, \text { all }|x|,|y| \leqslant M^{\prime} .
$$

Also, $L$ is an $N \times m$-matrix and we assume that there exist $M>0$ and $\widehat{a}_{M} \in L^{2}(T)$ such that

$$
(f(t, x)+L(v), x)_{\mathbb{R}^{N}} \geqslant 0 \quad \text { for a.a. } t \in T, \text { all }|x|=M, v \in C(t, x),
$$

and

$$
|f(t, x)|,|C(t, x)| \leqslant \widehat{a}_{M}(t) \quad \text { for a.a. } t \in T, \text { all }|x| \leqslant M .
$$

We set

$$
F(t, x)=f(t, x)+L(C(t, x)) \quad \forall(t, x) \in T \times \mathbb{R}^{N} .
$$

This multifunction satisfies hypotheses $H(F)_{4}$ while hypothesis $H_{0}$ is clearly satisfied (see Eq. 7.2). Also, we have

$$
\text { ext } F(t, x)=f(t, x)+\operatorname{ext} C(t, x) .
$$

Now, the control system (7.1) is equivalent to the following periodic differential inclusion:

$$
\left\{\begin{array}{l}
-u^{\prime}(t) \in \xi(t)|u(t)|^{p-2} u(t)+\partial|u(t)|+F(t, u(t)) \quad \text { for a.a. } t \in T \\
u(0)=u(b)
\end{array}\right.
$$

We know that the solution set $S_{c}$ of this multivalued system is nonempty (see Theorem 3.7) and in fact, we have

$$
S_{c} \in P_{k}\left(C\left(T ; \mathbb{R}^{N}\right)\right)
$$

(see Proposition 3.8). Furthermore, if $u \in S_{c}$ and $\varepsilon>0$, then we can find $u_{\varepsilon} \in$ $W^{1,2}\left((0, b) ; \mathbb{R}^{N}\right)$ such that

$$
\left\{\begin{array}{l}
-u_{\varepsilon}^{\prime}(t) \in \xi(t)\left|u_{\varepsilon}(t)\right|^{p-2} u_{\varepsilon}(t)+\partial\left|u_{\varepsilon}(t)\right|+f\left(t, u_{\varepsilon}(t)\right)+L\left(v_{\varepsilon}(t)\right) \quad \text { for a.a. } t \in T \\
u_{\varepsilon}(0)=u(0), v_{\varepsilon} \in L^{2}\left(T ; \mathbb{R}^{N}\right), v_{\varepsilon}(t) \in \operatorname{ext} C\left(t, u_{\varepsilon}(t)\right) \quad \text { for a.a. } t \in T
\end{array}\right.
$$

and $\left\|u-u_{\varepsilon}\right\|_{C\left(T ; \mathbb{R}^{N}\right)} \leqslant \varepsilon$ (see Theorem 6.1).

In fact, if $\xi(t) \geqslant \widehat{c}>0$ for all $t \in T$ and $1<p \leqslant 2$, then we can find $u_{\varepsilon} \in$ $W^{1,2}\left((0, b) ; \mathbb{R}^{N}\right)$ satisfying (7.3) with periodic boundary condition (that is, $u_{\varepsilon}(0)=u_{\varepsilon}(b)$ ) and

(see Theorem 6.3).

$$
\left\|u-u_{\varepsilon}\right\|_{C\left(T ; \mathbb{R}^{N}\right)} \leqslant \varepsilon
$$


Acknowledgments The authors wish to thank the three anonymous reviewers for their corrections and helpful remarks.

Open Access This article is distributed under the terms of the Creative Commons Attribution 4.0 International License (http://creativecommons.org/licenses/by/4.0/), which permits unrestricted use, distribution, and reproduction in any medium, provided you give appropriate credit to the original author(s) and the source, provide a link to the Creative Commons license, and indicate if changes were made.

\section{References}

1. Bader R, Papageorgiou NS. On the problem of periodic evolution inclusions of the subdifferential type. Z Anal Anwendungen. 2002;21:963-984.

2. Bressan A, Colombo G. Extensions and selections of maps with decomposable values. Studia Math. 1988;90:69-86.

3. Brézis H. Opérateurs Maximaux Monotones et Semi-Groupes de Contractions dans les Espaces de Hilbert. Amsterdam: North-Holland Publishing Co; 1973.

4. Egghe L. Stopping time techniques for analysts and probabilists, London Mathematical Society Lecture Note Series, Vol. 100. Cambridge: Cambridge University Press; 1984.

5. Frigon M. Systems of first order differential inclusions with maximal monotone terms. Nonlinear Anal. 2007;66:2064-2077.

6. Fryszkowski A. Continuous selections for a class of nonconvex multivalued maps. Studia Math. 1983;76:163-174.

7. Gasiński L., Papageorgiou NS. Nonlinear analysis. Boca Raton: Chapman \& hall/CRC; 2006.

8. Gasiński L, Papageorgiou NS. Exercises in analysis. Part 1. Cham: Springer; 2014.

9. Gasiński L, Papageorgiou NS. Exercises in analysis. Part 2: nonlinear analysis. Cham: Springer; 2014.

10. Hartman P. On boundary value problems for systems of ordinary, nonlinear, second order differential equations. Trans Amer Math Soc. 1960;96:493-509.

11. Hu S, Papageorgiou NS. Handbook of multivalued analysis. Vol I, theory. Dordrecht: Kluwer Academic Publishers; 1997.

12. Papageorgiou NS, Kyritsi S. Handbook of applied analysis. New York: Springer-Verlag; 2009.

13. Pavel NH. Differential equations associated with compact evolution generators. Differ Integr Equ. 1988;1:1:117-123.

14. Qin S, Xue X. Periodic solutions for nonlinear differential inclusions with multivalued perturbations. J Math Anal Appl. 2015;424:988-1005.

15. Zeidler E. Nonlinear functional analysis and its applications II/B. Nonlinear monotone operators. New York: Springer-Verlag; 1990. 